\title{
CHARACTERIZATION OF NAKAYAMA $m$-CLUSTER TILTED ALGEBRAS OF TYPE $A_{n}$
}

\author{
FAisAl AND InTAN Muchtadi-Alamsyah \\ Algebra Research Group \\ Institut Teknologi Bandung, \\ f1107101@students.itb.ac.id, ntan@math.itb.ac.id
}

\begin{abstract}
For any natural natural number $m$, the $m$-cluster tilted algebras are generalization of cluster tilted algebras. These class algebras are defined as the endomorphism of certain object in $m$-cluster category called $m$-cluster tilting object. Finding such object in the $m$-cluster category has become a combinatorial problem. In this article we characterize Nakayama $m$-cluster tilted algebras of type $A_{n}$ by geometric description given by Baur and Marsh.

Key words and Phrases: Cluster tilted algebras, cluster category, tilting object, Nakayama algebra

Abstrak. Untuk setiap bilangan asli $m$, aljabar teralih $m$-kluster adalah generalisasi dari aljabar teralih kluster. Kelas aljabar ini didefinisikan sebagai endomorfisma objek tertentu di kategori $m$-kluster yang disebut objek pengalih $m$-kluster. Mencari objek tersebut dalam kategori $m$-kluster dapat menjadi masalah kombinatorial. Dalam artikel ini dikarakterisasi aljabar Nakayama yang merupakan aljabar teralih $m$-kluster jenis $A_{n}$ berdasarkan deskripsi geometris yang diberikan oleh Baur dan Marsh.

Kata kunci: aljabar teralih kluster, kategori kluster, objek pengalih, aljabar Nakayama.
\end{abstract}

\section{INTRODUCTION}

Let $K$ be an algebraically closed field, and $Q$ a finite acyclic quiver with $n$ vertices. Let $\mathcal{D}^{b}(H)$ be a bounded derived category of $\bmod H$ where $H$ is a basic, finite dimensional hereditary algebra over $K$. We can assume $H$ as a path algebra $K Q$ of some quiver $Q$. The $m$-cluster category is the orbit category $C_{H}^{m}=\mathcal{D}^{b}(H) / \tau^{-1}[m]$ where $\tau$ is the Auslander-Reiten translation of $\mathcal{D}^{b}(H)$ and $[m]$ denotes $m$-th power of shift [1] in the derived category $\mathcal{D}^{b}(H)$. The $m$-cluster category is triangulated

2000 Mathematics Subject Classification: 16G70.

Received: 27-04-2015, revised: 07-08-2016, accepted: 07-08-2016. 
[5] and it is a Krull-Schmidt category [2]. These categories are generalization of cluster categories defined in [2] and independently [3] for the Dynkin type $A_{n}$ case.

In $m$-cluster category we consider a class of objects called $m$-cluster tilting objects. These objects have nice combinatorial properties. By definition, an object $T$ is an $m$-cluster tilting object if for any object $X$, we have $X \in$ add $T$ if only if $\operatorname{Ext}_{C_{H}^{m}}^{i}(T, X)=0$ for all $i \in\{1,2, \ldots, m\}$. The objects $T$ always have exactly $n$ indecomposable direct summands [7]. The endomorphism algebra $\operatorname{End}_{C_{H}^{m}}^{o p}(T)$ is called $m$-cluster tilted algebra.

In this paper we investigate $m$-Cluster Tilted Algebras $\left(m\right.$-CTA) of type $A_{n}$ which are Nakayama algebras. Nakayama algebra itself by its quiver is divided into two types, namely type $A_{n}$ and cyclic. In this paper we focus on $m$-CTAs which are Nakayama algebras of type $A_{n}$ and all possible relations as from [6] we have known all $m$-CTAs which are Nakayama algebras of type cyclic, see also [4]. In order to do this we use the geometric description of $m$-cluster category type $A_{n}$ in [1]. We will divide into three cases in the search of $m$-CTAs of type $A_{n}$. We divide these two cases based on the relationship between $m$ and $n$. The first case is when $m \geq n-2$, the second case is $m<n-2$.

This article is organized as follows. In Section 2 we describe the geometric description and the relations of Nakayama $m$-CTAs; in Section 3 we give a characterization of Nakayama $m$-CTA of cyclic type; in Section 4 we give a characterization of Nakayama $m$-CTA of acyclic type which will be divided into two cases.

\section{Geometric Description and Relations in Nakayama $m$-CTAs}

The geometric description of $m$-cluster category type $A_{n}$ in [1] briefly representing indecomposable objects and arrows of the AR-quiver of $m$-cluster category in a regular gon. The indecomposable object is described as a diagonal of a regular gon while an arrow between two indecomposable objects described as two diagonals that have a common endpoint. From this geometric description we can also see the relations of quivers of the $m$-CTAs of type $A_{n}$.

Let $\mathcal{P}_{m(n+1)+2}$ be $(m(n+1)+2)$-regular gon, $m, n \in \mathbb{N}$, where its corner points are numbered clockwise from 1 to $m(n+1)+2$. A diagonal $D$ of $\mathcal{P}_{m(n+1)+2}$ can be denoted as a pair $(i, j)$. Consequently, the diagonal $(i, j)$ is the diagonal $(j, i)$. We said a diagonal $D$ of $\mathcal{P}_{m(n+1)+2}$ is an $m$-diagonal if $D$ divide $\mathcal{P}_{m(n+1)+2}$ into two parts that is $(m j+2)$-gon and $(m(n-j)+2)$-gon where $j=1,2, \ldots,\left\lceil\frac{n}{2}\right\rceil$. For $i \neq j$, an arc $D_{i j}$ of $\mathcal{P}_{m(n+1)+2}$ is a part of boundary that connect $i$ to $j$ clockwise. Note that if $j$ is a clockwise direct neighbor of $i$ then arc $D_{i j}$ is an edge $i j$ of $\mathcal{P}_{m(n+1)+2}$. We always have two arcs $D_{i j}, D_{j i}$. Let $\Gamma_{A_{n}}^{m}$ be a quiver with the vertices are all $m$-diagonals of polygon $\mathcal{P}_{m(n+1)+2}$ while arrows obtained in the following way: suppose $D=(i, j)$ and $D^{\prime}=\left(i, j^{\prime}\right)$ are $m$-diagonals which have a common vertex $i$ in $\mathcal{P}_{m(n+1)+2}$ then there is an arrow from $D$ to $D^{\prime}$ if $D, D^{\prime}$ 
together with arc from $j$ to $j^{\prime}$ form $(m+2)$-gon in $\mathcal{P}_{m(n+1)+2}$ and $D$ can be rotated clockwise to $D^{\prime}$ about the common endpoint $i$.

Using this regular gon we can easily make a quiver of an $m$-CTA. The set of indecomposable objects of a tilting object of $m$-cluster category of type $A_{n}$ can be identified as the set of maximal $m$-diagonals in $\mathcal{P}_{m(n+1)+2}$ and the number of direct summands of this object is always $n$. Such a set is called an $(m+2)$-angulation of $\mathcal{P}_{m(n+1)+2}$. By definition, we can conclude that if $X$ and $Y$ are $m$-diagonals of a tilting object $T$ that has a common endpoint then there is a path from $T_{X}$ and $T_{Y}$ in the Auslander-Reiten(AR) quiver of $m$-cluster category where $T_{X}$ and $T_{Y}$ are indecomposable objects associated to $X$ and $Y$. It is clear that the composition of the arrows in this path is not zero. If there is no $m$-diagonal between $X$ and $Y$ in $\mathcal{P}_{m(n+1)+2}$ then the composition of irreducible maps from $T_{X}$ to $T_{Y}$ does not pass through another indecomposable object which is a direct summand of a tilting object $T$. It means that there is an arrow from the point corresponding to $X$ and $Y$ in the quiver of $m$-CTA $\operatorname{End}^{o p}(T)$.

By the above argument we can define a quiver of an $m$-CTA independently from $(m+2)$-angulation of $\mathcal{P}_{m(n+1)+2}$. Let $T=\left\{T_{1}, T_{2}, \ldots, T_{n}\right\}$ be an $(m+2)$ angulation. Define a quiver $Q_{T}$ as follows: The vertices of $Q_{T}$ are the numbers $1,2, \ldots, n$ which are in bijective correspondence with the $m$-diagonals $T_{1}, T_{2}, \ldots, T_{n}$. Given two vertices $a, b$ of $Q_{T}$, there is an arrow from $a$ to $b$ if

(i) $T_{a}$ and $T_{b}$ have a common point in $\mathcal{P}_{m(n+1)+2}$,

(ii) there is no $m$-diagonal of $T$ between $T_{a}$ and $T_{b}$ and

(iii) $T_{a}$ can be rotated clockwise to $T_{b}$ at the common endpoint.

Our first lemma characterize the possible forms of two $m$-diagonals in polygon $\mathcal{P}_{m(n+1)+2}$, correspond to a path of length two in the quiver of an $m$-CTA. We have the following easy lemma.

Lemma 2.1. Let $H=E n d^{o p}(T)$ be an $m$-CTA with $T$ is an $m$-cluster tilting object of $\mathcal{C}_{A_{n}}^{m}$. If $x \rightarrow y \rightarrow z$ is a path of length two in $Q_{H}$ and $T_{x}, T_{y}, T_{z}$ respectively are $m$-diagonals correspond to points $x, y, z$ then

(1) $T_{x}=\left(x_{1}, x_{2}\right), T_{y}=\left(x_{2}, x_{3}\right), T_{z}=\left(x_{3}, x_{4}\right)$ with $x_{4}$ in arc $D_{x_{3} x_{1}}$

$$
\text { or }
$$

(2) $T_{x}=\left(x_{1}, x_{2}\right), T_{y}=\left(x_{2}, x_{3}\right), T_{z}=\left(x_{2}, x_{4}\right)$ with $x_{4}$ in arc $D_{x_{3} x_{2}}$,

where $x_{i} \neq x_{j}$ if $i \neq j$.

Proof. Let $T_{x}=\left(x_{1}, x_{2}\right)$. Since there is an arrow from $x$ to $y$ then $T_{x}$ and $T_{y}$ have a common endpoint. Without loss of generality, suppose $T_{y}=\left(x_{2}, x_{3}\right)$. Since there is an arrow from $y$ to $z$ then $T_{y}$ and $T_{z}$ have a common endpoint. If $x_{3}$ is a common endpoint of $T_{y}$ and $T_{z}$ then $T_{z}=\left(x_{3}, x_{4}\right)$ where $x_{4}$ in $\operatorname{arc} D_{x_{1} x_{3}}$, otherwise $T_{z}$ will cross $T_{x}$. If $x_{2}$ is a common endpoint of $T_{y}$ and $T_{z}$ then $T_{z}=\left(x_{2}, x_{4}\right)$ where $x_{4}$ in $\operatorname{arc} D_{x_{3} x_{2}}$.

Let $Q$ be a finite quiver without cycle and $H=K Q / \mathcal{I}$ where $\mathcal{I}$ is an admissible ideal of $K Q$. If $Q$ is not connected then the algebra $H$ is not connected. Indeed 
let $\mathcal{Q}$ be the collection of maximal connected subquivers of $Q$. It can be shown that $H=\prod_{Q^{\prime} \in \mathcal{Q}} K Q^{\prime} / \mathcal{I}^{\prime}$ where $\mathcal{I}^{\prime}$ is an ideal of $Q^{\prime}$, but then $H$ is a finite direct product of some algebras. Hence, $H$ is not connected.

In order to know the condition of an $(m+2)$-angulation such that the quiver of $m$-cluster tilted algebra is connected, we have the following easy lemma.

Lemma 2.2. Let $T$ be an $(m+2)$-angulation of $\mathcal{P}_{m(n+1)+2}$. The graph generated by the diagonals in $T$ is connected if only if the quiver $Q_{T}$ is connected.

Let $X=\left(x_{1}, x_{2}\right)$ be a diagonal of $\mathcal{P}_{m(n+1)+2}$. We may assume $x_{2}>x_{1}$. Define the length of diagonal $X$ to be the $\min \left\{x_{2}-x_{1}, m(n+1)+2+x_{1}-x_{2}\right\}$. Thus, the length of $X$ is equal to the minimum of the number of sides between arc $D_{x_{1} x_{2}}$ and $D_{x_{2} x_{1}}$. An $m$-diagonal $X$ of $\mathcal{P}_{m(n+1)+2}$ is said to be short if its length

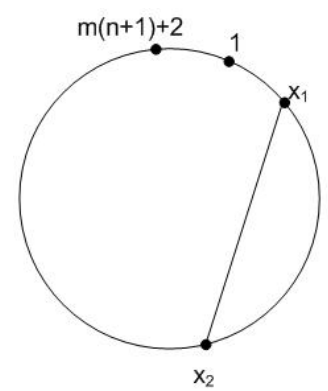

FiguRE 1. short $m$-diagonal

is minimal, that is of length $m+1$. An $m$-diagonal $X$ is short if only if there is no $m$-diagonal whose endpoints are in smaller polygon divided by $X$.

Lemma 2.3. Let $T$ be an $(m+2)$-angulation of $\mathcal{P}_{m(n+1)+2}$ with $n \geq 3$. If $Q_{T}$ is cyclic then all $m$-diagonals in $T$ are short.

Proof. Let $X$ be an $m$-diagonal of $T$ which is not short. Without loss of generality, let $X=\left(1, x_{1}\right)$ and $X$ has length which is minimal among the diagonals in $T$ which are not short. First, assume that $x_{1} \leq \frac{m(n+1)+2}{2}$. The diagonal $X$ will divide $\mathcal{P}_{m(n+1)+2}$ into two smaller polygons $P_{1}$ and $P_{2}$ with $P_{1}$ is the smallest polygon (see Figure 2). Since $X$ is not short and $T$ is maximal, there exists an $m$-diagonal of $T$ whose endpoints in $\operatorname{arc} D_{x_{1} x_{2}}$. By the same argument we also have another $m$-diagonal of $T$ which divides the polygon $P_{2}$. We then have that all $m$-diagonals in $P_{1}$ are short by the minimality of $X$. Since $Q_{T}$ is connected there exists a short $m$-diagonal $X_{1}$ of $T$ in $P_{1}$ that adjacent to $X$. We may assume that $X_{1}=(1, b)$. Now there exists a short $m$-diagonal that adjacent to $X_{1}$, namely $X_{2}$. By the same argument we have a collection of short $m$-diagonals $X_{1}=\left(1, a_{1}\right), X_{2}=$ $\left(a_{1}, a_{2}\right) \ldots, X_{k}=\left(a_{k-1}, a_{k}\right)$ where all of these are in $P_{1}$ and maximal with respect 


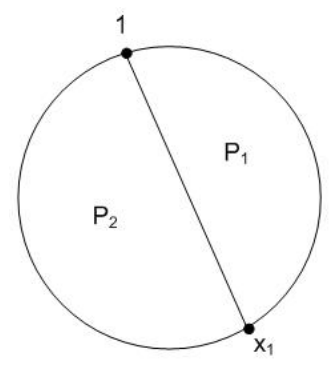

Figure 2. $m$-diagonal $X$

to this property. It follows that $x_{k}=x_{1}$, otherwise there is no arrow which target is $X_{k}$ in $Q_{T}$. We describe this situation in the following figure

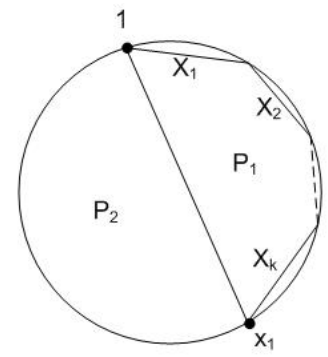

FiguRe $3 . \quad m$-diagonals in $P_{1}$

But now we have a path $X_{1} \rightarrow X \rightarrow X_{k}$ in $Q_{T}$. So there can be no further $m$-diagonals adjacent to $X$, which is a contradiction.

If $x_{1}>\frac{m(n+1)+2}{2}$ we get similar proof for $P_{2}$ since in this case $P_{2}$ becomes the smallest polygon divided by $X$.

Lemma 2.3 gives us a characterization of $m$-cluster tilting object such that the corresponding $m$-CTA is a Nakayama algebra of cyclic type. We will find all $m$-cluster tilting objects in this form in the next section. Now we look at the configuration of an $(m+2)$-angulation $T$ which $Q_{T}$ is of $A_{n}$ type.

Lemma 2.4. Let $T$ be an $(m+2)$-angulation of $\mathcal{P}_{m(n+1)+2}$ with $n \geq 3$. If $Q_{T}$ is of $A_{n}$ type then

$$
T=T_{C} \cup T_{\alpha_{1}} \cup T_{\alpha_{2}} \cup \cdots \cup T_{\alpha_{r-1}}
$$


for some $r \geq 2$ where (up to rotation) $T_{C}=\left\{\left(1, x_{1}\right),\left(x_{1}, x_{2}\right), \ldots,\left(x_{r-1}, x_{r}\right)\right\}$ and all $m$-diagonals in $T_{C}$ are short,

$$
\begin{array}{r}
T_{\alpha_{1}}=\left\{\left(x_{1}, y_{11}\right),\left(x_{1}, y_{12}\right), \ldots,\left(x_{1}, y_{1 j_{1}}\right)\right\}, j_{1} \geq 0 \\
T_{\alpha_{2}}=\left\{\left(x_{2}, y_{21}\right),\left(x_{1}, y_{22}\right), \ldots,\left(x_{1}, y_{2 j_{2}}\right)\right\}, j_{2} \geq 0 \\
\vdots \\
T_{\alpha_{r-1}}=\left\{\left(x_{r-1}, y_{r-1,1}\right),\left(x_{1}, y_{r-1,2}\right), \ldots,\left(x_{r-1}, y_{r-1, j_{r-1}}\right)\right\}, j_{r-1} \geq 0 \\
\text { with } y_{11}<y_{12}<\cdots<y_{1 j_{1}}<y_{21}<\cdots<y_{2 j_{2}}<\cdots<y_{n-1, j_{n-1}} .
\end{array}
$$

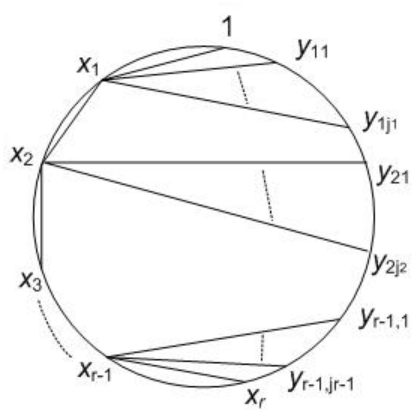

Figure 4. $(m+2)$-angulation of $T$ with $Q_{T}=A_{n}$

Proof. Let $\left(1, x_{1}\right)$ be an $m$-diagonal of $\mathcal{P}_{m(n+1)+2}$ correspond to a source in $Q_{T}$. We claim that $\left(1, x_{1}\right)$ is short. If $\left(1, x_{1}\right)$ is not short then either there is an $m$ diagonal $\left(x_{1}, t\right)$ with $t>x_{1}$ or there is an $m$ diagonal $(1, u)$ with $u>x_{1}$ (see Figure $5)$. Consider the first case, if there is an $m$-diagonal $\left(x_{1}, t\right)$, we chose $t$ maximal
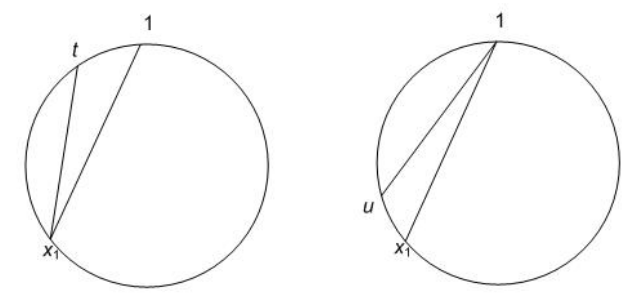

FiguRE 5. $m$-diagonals $\left(x_{1}, t\right)$ and $(1, u)$

such that $t>x_{1}$. Then we have an arrow $\left(x_{1}, t\right) \rightarrow\left(1, x_{1}\right)$, but it contradicts that $\left(1, x_{1}\right)$ is a source. Second case, if there is an $m$-diagonal $(1, u)$ we chose $u$ minimal such that $u>x_{1}$. Since $\left(1, x_{1}\right)$ is not short, there is either an $m$-diagonal $\left(x_{1}, a\right)$ 
with $1<a<x_{1}$ or an $m$-diagonal $(1, b)$ with $1<b<x_{1}$. We may assume that $a$ is minimal and $b$ maximal. If there is a diagonal $\left(x_{1}, a\right)$ then there is an arrow $(1, b) \rightarrow\left(x_{1}, a\right)$. It contradicts the fact that there is also an arrow $\left(1, x_{1}\right) \rightarrow(1, u)$. So we can assume that there is a diagonal $(1, b)$. It follows that there is an arrow $(1, b) \rightarrow\left(1, x_{1}\right)$. This is a contradiction since $\left(1, x_{1}\right)$ is a source. Therefore $\left(1, x_{1}\right)$ is short, this proves our claim.

Let $\left(1, x_{1}\right) \rightarrow\left(x_{1}, z\right)$ be the arrow starting in $\left(1, x_{1}\right)$ then $z>1$. Now there are two cases, either $\left(x_{1}, z\right)$ is short or $\left(x_{1}, z\right)$ is not short.

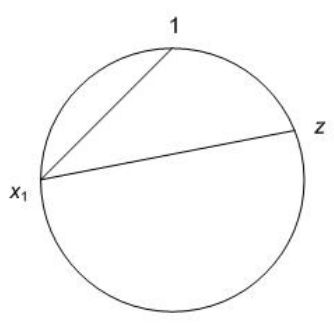

FiguRE $6 . \quad m$-diagonal $\left(x_{1}, z\right)$

(1) $\left(x_{1}, z\right)$ is short.

If $T_{\alpha}=\left(x_{1}, z\right)$ is short then $\operatorname{arc} D_{z x_{1}}$ together with $T_{\alpha}$ is a smaller polygon divided by $\left(x_{1}, z\right)$. Hence, there is no $m$-diagonal with endpoints in arc $D_{z x_{1}}$. We also have that there is no $m$-diagonal $\left(x_{1}, y\right)$ with $1<y<z$ since otherwise the arrow $\left(1, x_{1}\right) \rightarrow\left(x_{1}, z\right)$ will not exist.

(2) $\left(x_{1}, z\right)$ is not short.

If $\left(x_{1}, z\right)$ is not short then there is no $m$-diagonal $(z, v)$ with $1<v<z$. Indeed, assume to the contrary that there is an $m$-diagonal $(z, v)$ with $1<v<z$. It follows that there is no $m$-diagonal $\left(x_{1}, u\right)$ for $z<u<x_{1}$ since otherwise there is also an arrow $\left(x_{1}, z\right) \rightarrow\left(x_{1}, u\right)$. If there is an $m$ diagonal $(z, l)$ for $z<l<x_{1}$, and choose $z$ maximal, then there is an arrow $(z, l) \rightarrow\left(x_{1}, z\right)$, a contradiction. Therefore there is no $m$-diagonal with endpoints in $\operatorname{arc} D_{z x_{1}}$. This is a contradiction since $\left(x_{1}, z\right)$ is not short. Hence there is no diagonal $(z, v)$. Therefore arc $D_{1 z}$ together with $\left(1, x_{1}\right)$ and $\left(x_{1}, z\right)$ forms an $(m+2)$-gon.

We describe condition 1 and 2 respectively as follows
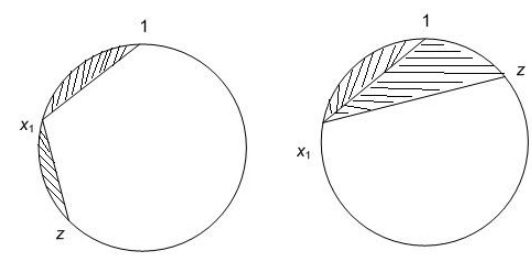

where the shaded polygons are $m+2$-gons and hence there is no $m$-diagonal in these polygons. Now we perform same analysis by consider the arrow starting at $\left(x_{1}, z\right)$. 
Indeed, in case $\left(x_{1}, z\right)$ is short then the arrow starting at $\left(x_{1}, z\right)$ is $\left(x_{1}, z\right) \rightarrow(z, w)$ with $1<w<z$. In case $\left(x_{1}, z\right)$ is not short then the arrow starting at $\left(x_{1}, z\right)$ is $\left(x_{1}, z\right) \rightarrow\left(x_{1}, w\right)$ with $1<w<x$. We have similar case for the third $m$-diagonal from the source which adjacent to $\left(x_{1}, z\right)$. There are again two cases to consider, that is either this $m$-diagonal is short or not short. These two cases will be similar to the condition 1 and 2 above. We complete the proof by induction using the fact that the the next $m$-diagonal adjacent to the previous have two possibilities like condition 1 and 2 .

Two cases in Lemma 2.1 hold for any path of length two in the quiver of $m$-CTAs of type $A_{n}$. For both cases the picture is as follows

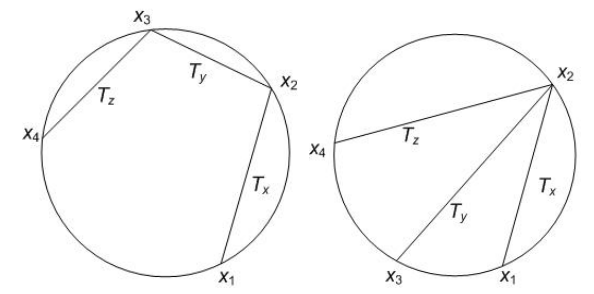

FiguRE 7. $m$-diagonals correspond a path of length two

Using the above lemma we can conclude that each path of length two in the quiver of $m$-CTAs of type $A_{n}$ is one of these two cases.

Now we will see the composition of paths of length two in End $(T) \cong K Q / \mathcal{I}$ for both cases. We have the following facts.

Lemma 2.5. Let $T=T_{1} \oplus T_{2} \oplus \cdots \oplus T_{n}$ be an $m$-cluster tilting object of $\mathcal{C}_{A_{n}}^{m}$ and $Q$ be a quiver of $m$-CTA $\operatorname{End}^{o p}(T)$. Suppose $i \stackrel{\alpha}{\rightarrow} j \stackrel{\beta}{\rightarrow} k$ is a path of length two in $Q$ corresponding to the $m$-diagonals $T_{i}, T_{j}, T_{k}$ in $\mathcal{P}_{m(n+1)+2}$.

(1) If $T_{i}=\left(x_{1}, x_{2}\right), T_{j}=\left(x_{2}, x_{3}\right), T_{k}=\left(x_{3}, x_{4}\right)$ with $x_{4}$ in arc $D_{x_{3} x_{1}}$ then the composition $i \stackrel{\alpha}{\rightarrow} j \stackrel{\beta}{\rightarrow} k$ in $\operatorname{End}^{o p}(T)$ is zero.

(2) If $T_{x}=\left(x_{1}, x_{2}\right), T_{y}=\left(x_{2}, x_{3}\right), T_{z}=\left(x_{2}, x_{4}\right)$ with $x_{4}$ in arc $D_{x_{3} x_{2}}$ then the composition $i \stackrel{\alpha}{\rightarrow} j \stackrel{\beta}{\rightarrow} k$ in $\operatorname{End}^{o p}(T)$ is not zero.

Proof. See [4].

Now we can identify the relation of connected Nakayama $m$-cluster tilted algebras using Lemma 2.3, 2.4 and 2.5.

Theorem 2.6. Let $H=K Q / \mathcal{I}$ be a connected Nakayama m-cluster tilted algebra of $\mathcal{C}_{A_{n}}^{m}$. An ideal $\mathcal{I}$ of $H$ is generated by a relation of paths of length two.

Proof. If $Q$ is cyclic then by Lemma $2.3, Q=Q_{T}$ where $T$ is an $(m+2)$-angulation such that all $m$-diagonals in $T$ are short. Therefore, every path of length two in 
$Q_{T}$ is in case 1 of Lemma 2.1. By Lemma 2.5 all paths of length two is zero. If $Q$ is of type $A_{n}$ then by Lemma 2.4 every path of length two is either case one or case two of Lemma 2.5. It remains to prove that every path $\mathbb{P}=\alpha_{1} \alpha_{2} \ldots \alpha_{\ell}$ with $\ell \geq 3$ is not zero in $H$ if every subpath of $\mathbb{P}$ is not zero in $H$. It follows that every subpath of length two in $\mathbb{P}$ is case two of Lemma 2.5. We may assume that $T_{\alpha_{1}}=(1, m r+2)$ with $1 \leq r<n$ whose common endpoint with $T_{\alpha_{2}}$ and $T_{\alpha_{3}}$ is 1 . Hence, $T_{\alpha_{j}}=\left(1, m r_{j}+2\right)$ for every $j \geq 2$ with $r<r_{i}<r_{i+1}$ for all $i$. We have that $T_{\alpha_{1}}, T_{\alpha_{2}}, \ldots, T_{\alpha_{\ell}}$ will be in the subquiver of $\Gamma_{A_{n}}^{m}$ as in Figure 8. Since the

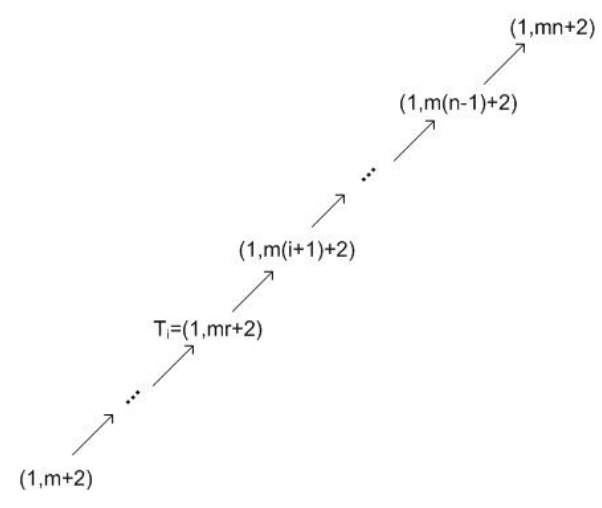

FigURE 8. subquiver of $\Gamma_{A_{n}}^{m}$

composition of irreducible morphism $T_{\alpha_{1}} \rightarrow T_{\alpha_{2}} \rightarrow \cdots \rightarrow_{\alpha_{\ell}}$ is not zero in $m$-cluster category, we conclude that $\alpha_{1} \alpha_{2} \ldots \alpha_{\ell}$ not zero in $H$. This finishes the proof.

\section{3. m-CTAs which are Nakayama Algebra of Cyclic Type}

In this section we will show that $m$-CTAs which are Nakayama algebras of cyclic type only occur if $m=n-2$. It means that there is no $m$-CTA whose quiver is cyclic when $m \neq n-2$. In addition, in $m$-CTA there is only one possibility relation that is relations of paths of length two. More generally, $m$-CTAs which have cyclic quivers have been stated by Murphy in [6]. However, in this section we explain how to characterize $m$-CTAs which quivers are cyclic by using geometric description in [1]. The results in this section have been proved in [4]. We state again here with more structured proofs.

We show that if $T=T_{1} \oplus T_{2} \oplus \cdots \oplus T_{n}$ then $T$ is a $m$-cluster tilting object for $m \geq n+2$ where $T_{i}$ 's are $m$-diagonals described in Proposition 3.1. The quivers of $m$-CTAs $\operatorname{End}^{o p}(T)$ have different forms for each case $m=n-2$ and $m>n-2$. Indeed, for $1 \leq i \leq n-1$ diagonals $T_{i}$ and $T_{i+1}$ have a common endpoint in $\mathcal{P}_{m(n+1)+2}$ for $m \geq n-2$. It means that for every $i$, we have an arrow $i \rightarrow i+1$ in 
the quiver of $\operatorname{End}^{o p}(T)$. Now consider $m$-diagonals $T_{n}=(3 m-(n-5), 2 m-(n-4))$ and $T_{1}=(1, m+2)$. If $m=n-2$ then $T_{n}=(2 m+3, m+2)$. Hence, $T_{n}$ and $T_{1}$ have a common endpoint $(m+2)$ in $\mathcal{P}_{m(n+1)+2}$. Therefore there exists an arrow $n \rightarrow 1$ in quiver of $\operatorname{End}^{o p}(T)$. Thus, for $m=n-2$ the quiver of $m$-cluster tilted algebra $\operatorname{End}^{o p}(T)$ is Figure 9.

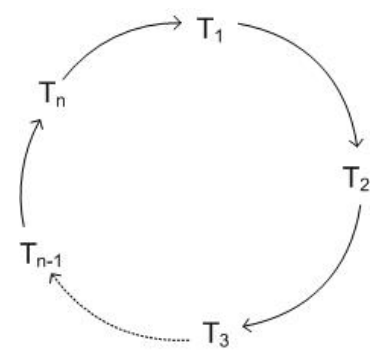

FiguRE 9. Quiver of $\operatorname{End}^{o p}(T)$ for $m=n-2$

Proposition 3.1. Let $\mathcal{C}_{A_{n}}^{m}=\mathcal{D}^{b}\left(K A_{n}\right) / F_{m}$, where $F_{m}=\tau^{-1}[m]$ and $m=n-2$. Suppose that $T_{1}=(1, m+2), T_{2}=(1, n m+2)$ and for $3 \leq i \leq n$,

$$
T_{i}=((n-(i-2)) m-(i-4),(n-(i-3)) m-(i-5))
$$

then

(1) $T_{1}, T_{2}, \ldots, T_{n}$ are $m$-diagonals of $\mathcal{P}_{m(n+1)+2}$.

(2) $T=T_{1} \oplus T_{2} \oplus \cdots \oplus T_{n}$ is an $m$-cluster tilting object.

(3) $m$-cluster tilted algebra End $d^{o p}(T)$ is isomorphic to $K Q / \mathcal{I}$ where $Q$ is cyclic with $n$ vertices and $\mathcal{I}$ is an ideal generated by all paths of length two.

Proof. It is clear that if $T_{1}=(1, m+2), T_{2}=(1, n m+2)$ and for $3 \leq i \leq n$,

$$
T_{i}=((n-(i-2)) m-(i-4),(n-(i-3)) m-(i-5))
$$

then $T_{1}, T_{2}, \ldots, T_{n}$ are $m$-diagonals of $\mathcal{P}_{m(n+1)+2}$. For $i=n$ we have that $T_{n}=$ $((n-(n-2)) m-(n-4),(n-(n-3)) m-(n-5))=(3 m-(n-5), 2 m-(n-$ $4)$ ). Consider $m$-diagonals $T_{1}, T_{2}, \ldots, T_{n}$ in $\mathcal{P}_{m(n+1)+2}$, see Figure 10. Because $T_{1}, T_{2} \ldots, T_{n}$ are not crossing each other then $T$ is an $m$-cluster tilting object. Let $Q$ be a quiver of $m$-cluster tilted algebra $\operatorname{End}^{o p}(T)$, then there is only one arrow $i \rightarrow i+1$ for every $1 \leq i \leq n-1$. Since $m=n-2$, we obtain that $T_{n}=(2 m+3, m+2)$ and $T_{1}=(1, m+2)$ have a common endpoint. Consequently, there is exactly one arrow $n \rightarrow 1$ in $Q$. It means that $Q$ is a cyclic quiver with $n$ vertices. By Lemma 2.5 the composition of all paths of length two is zero.

Next we show that the $m$-CTA of type $A_{n}$ whose quiver is cyclic is the algebra stated in Proposition 3.1. 


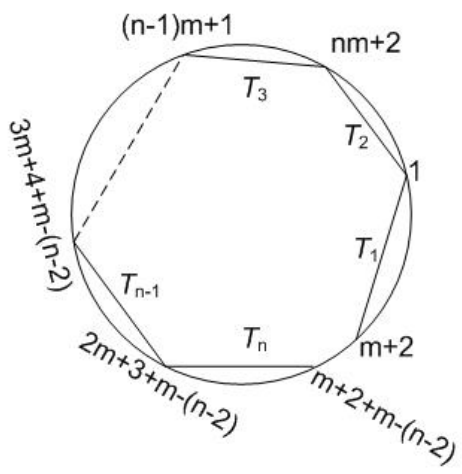

Figure 10. $m$-diagonals $T_{1}, T_{2}, \ldots, T_{n}$

Proposition 3.2. If $T$ is an m-cluster tilting object of $m$-cluster category $\mathcal{C}_{A_{n}}^{m}$ such that the quiver of $m$-cluster tilted algebra $\operatorname{End}^{o p}(T)$ is connected and cyclic, then $m=n-2$. Moreover, End ${ }^{o p}(T)=K Q / \mathcal{I}$ with $\mathcal{I}$ an ideal generated by all paths of length two.

Proof. Let $Q$ be a quiver of $m$-cluster tilted algebra $\operatorname{End}^{o p}(T)$. Suppose $T=$ $T_{1} \oplus T_{2} \oplus \cdots \oplus T_{n}$,we may assume $\left\{T_{1}, T_{2}, \ldots, T_{n}\right\}$ is a set of maximal non-crossing $m$-diagonals in $(m(n+1)+2)$-gon $\mathcal{P}_{m(n+1)+2}$. Assume that $Q_{0}=\left\{T_{1}, T_{2}, \ldots, T_{n}\right\}$ the set of vertices of $Q$, and the set of arrows $Q_{1}=\left\{\alpha_{1}, \alpha_{2}, \ldots, \alpha_{n-1}, \alpha_{n}\right\}$ with $\alpha_{i}: T_{i} \rightarrow T_{i+1}$ for every $i \in\{1,2, \ldots, n-1\}$ and $\alpha_{n}: T_{n} \rightarrow T_{1}$. Consider any path of length two $T_{p} \rightarrow T_{q} \rightarrow T_{r}$ in $Q$. By Lemma $2.3 T_{q}, T_{r}, T_{s}$ are short. It follows that $T_{q}=\left(x_{1}, x_{2}\right), T_{r}=\left(x_{2}, x_{3}\right), T_{s}=\left(x_{3}, x_{4}\right)$ can be described as in Figure 11. By applying the above argument, the picture of $m$-diagonals $T_{1}, T_{2}, \ldots, T_{n}$ in

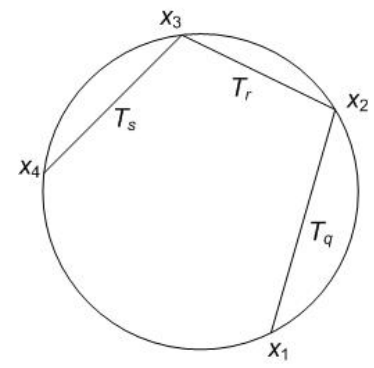

FiguRE 11. $m$-diagonals correspond to $T_{q}, T_{r}$ and $T_{s}$

$\mathcal{P}_{m(n+1)+2}$ is Figure 12 . 


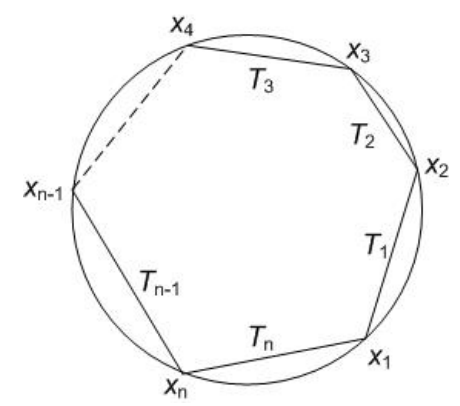

FiguRE 12. $m$-diagonals $T_{1}, T_{2}, \ldots, T_{n}$ for $m=n-2$

Since all $T_{i}$ are short then the length of $T_{i}$ is $m+1$. Consequently, we have the equation

$$
\underbrace{(m+1)+(m+1)+\cdots+(m+1)}_{n}=m(n+1)+2 .
$$

Therefore,

$$
(m+1) n=m(n+1)+2 \Leftrightarrow n=m+2
$$

For the last statement we apply Lemma 3.1.

Example 3.3. Let $m=4$ and $n=6$ then $m(n+1)+2=4(6+1)+2=30$. Consider 30-gon $\mathcal{P}_{30}$, let $T_{1}=(1,6), T_{2}=(1,26), T_{3}=(26,21), T_{4}=(21,16), T_{5}=(16,11)$ and $T_{6}=(11,6)$ then $T=T_{1} \oplus T_{2} \oplus T_{3} \oplus T_{4} \oplus T_{5} \oplus T_{6}$ is a 4-cluster tilting object. The picture of $\mathcal{P}_{30}$ together with the six $m$-diagonals is

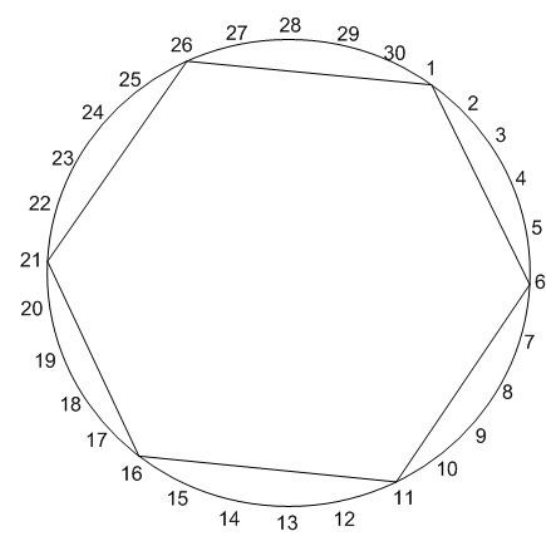

FiguRE 13. $(m+2)$-angulation $T$ for $m=4$ and $n=6$ 


\section{4. m-CTAs which are Nakayama Algebras with ACyclic Quivers}

In this section we will characterize $m$-CTA which are Nakayama algebras whose quivers are connected acyclic. In other words, we find $m$-cluster tilting objects $T=T_{1} \oplus T_{2} \oplus \cdots \oplus T_{n}$ such that $\operatorname{End}^{o p}(T) \cong K Q / \mathcal{I}$ where $Q$ is

$$
T_{1} \stackrel{\alpha_{1}}{\longrightarrow} T_{2} \stackrel{\alpha_{2}}{\longrightarrow} T_{3} \rightarrow \cdots \rightarrow T_{n-1} \stackrel{\alpha_{n-1}}{\longrightarrow} T_{n} .
$$

Throughout, $Q$ is assumed to be the above quiver, unless otherwise specified.

We will also observe the relation in this type of $m$-CTA. To do this we divide into two cases correspond to $m$ and $n$. These three cases are $m \geq n-2$ and $m<n-2$.

The following is the list of $m$-diagonals in $\mathcal{P}_{m(n+1)+2}$.

TABLE $1 . m$-diagonals

\begin{tabular}{|c|c|c|c|c|}
\hline$(1,-)$ & $(n m+2,-)$ & $((n-1) m+1,-)$ & $((n-2) m,-)$ & $((n-3) m-1,-)$ \\
\hline$m+2$ & 1 & $n m+2$ & $(n-1) m+1$ & $(n-2) m$ \\
$2 m+2$ & $m+1$ & $(n+1) m+2$ & $n m+1$ & $(n-1) m$ \\
$3 m+2$ & $2 m+1$ & $m$ & $(n+1) m+1$ & $n m$ \\
$4 m+2$ & $3 m+1$ & $2 m$ & $m-1$ & $(n+1) m$ \\
$\vdots$ & $\vdots$ & $\vdots$ & $\vdots$ & $\vdots$ \\
$n m+2$ & $(n-1) m+1$ & $(n-2) m$ & $(n-3) m-1$ & $(n-4) m-2$ \\
\hline
\end{tabular}

\begin{tabular}{|c|c|c|c|c|}
\hline$((n-4) m-2,-)$ & $((n-5) m-3,-)$ & $\ldots$ & $((n-i) m-(i-2),-)$ & $((n-(i+1)) m-(i-1),-)$ \\
\hline$(n-3) m-1$ & $(n-4) m-2$ & $\ldots$ & $(n-(i-1)) m-(i-3)$ & $(n-i) m-(i-2)$ \\
$(n-2) m-1$ & $(n-3) m-2$ & $\ldots$ & $(n-(i-2)) m-(i-3)$ & $(n-(i-1)) m-(i-2)$ \\
$(n-1) m-1$ & $(n-2) m-2$ & $\ldots$ & $(n-(i-3)) m-(i-3)$ & $(n-(i-2)) m-(i-2)$ \\
$n m-1$ & $(n-1) m-2$ & $\ldots$ & $\vdots$ & $\vdots$ \\
$(n+1) m-1$ & $n m-2$ & $\ldots$ & $n m-(i-3)$ & $(n-1) m-(i-2)$ \\
$m-3$ & $(n+1) m-2$ & $\ldots$ & $(n+1) m-(i-3)$ & $n m-(i-2)$ \\
$2 m-3$ & $m-4$ & $\ldots$ & $m-(i-1)$ & $(n+1) m-(i-2)$ \\
$\vdots$ & $\vdots$ & $\vdots$ & $\vdots$ & $\vdots$ \\
$(n-5) m-3$ & $(n-6) m-5$ & $\ldots$ & $(n-(i+1)) m-(i-1)$ & $(n-(i+2)) m-i$ \\
\hline
\end{tabular}


From Table 1 we take $m$-diagonals which will be used as a direct summand of an $m$-cluster tilting object such that the quiver of $m$-CTA is $A_{n}$. The following table lists some $m$-diagonals which will be used for our $m$-cluster tilting object.

TABLE 2. $m$-diagonals of $m$-cluster tilting objects

\begin{tabular}{|c|c|}
\hline$X_{1,1}=(1,2 m+2)$ & $X_{1,2}=(n m+2,2 m+1)$ \\
$X_{2,1}=(1,3 m+2)$ & $X_{2,2}=(n m+2,3 m+1)$ \\
$X_{3,1}=(1,4 m+2)$ & $X_{3,2}=(n m+2,4 m+1)$ \\
$\vdots$ & $\vdots$ \\
$X_{n-2,1}=(1,(n-1) m+2)$ & $X_{n-2,2}=(n m+2,(n-1) m+1)$ \\
\hline
\end{tabular}

\begin{tabular}{|c|c|c|}
\hline$X_{1,3}=((n-1) m+1,2 m)$ & $\ldots$ & $X_{1, i}=((n-(i-2)) m-(i-4), 2 m-(i-3))$ \\
$X_{2,3}=((n-1) m+1,3 m)$ & $\ldots$ & $X_{2, i}=((n-(i-2)) m-(i-4), 3 m-(i-3))$ \\
$X_{3,3}=((n-1) m+1,4 m)$ & $\ldots$ & $X_{3, i}=((n-(i-2)) m-(i-4), 4 m-(i-3))$ \\
$\vdots$ & $\vdots$ & $\vdots$ \\
$X_{n-3,3}=((n-1) m+1,(n-2) m)$ & $\ldots$ & $X_{n-i, i}=((n-(i-2)) m-(i-4),(n-i+1) m-(i-3))$ \\
\hline
\end{tabular}

Throughout, for every $1 \leq i \leq n, T_{i}$ is assumed to be the $m$-diagonal described in Proposition 3.1.

4.1. Case $m \geq n-2$.

Recall that $T_{1}=(1, m+2), T_{2}=(1, n m+2)$ and for $3 \leq i \leq n-t$ we have

$$
T_{i}=((n-(i-2)) m-(i-4),(n-(i-3)) m-(i-5)) .
$$

We have that all $m$-diagonals in the set $T=\left\{T_{1}, T_{2}, \ldots T_{n-1}, T_{n}\right\}$ are short. In the case $m=n-2$ the quiver of $Q_{T}$ is a cyclic quiver and every path of length of two is a relation in the corresponding $m$-CTA. We will prove that there is no $m$-CTA whose quiver is $A_{n}$ and every path of length two is zero in the case $m=n-2$. But in the case $m>n-2$ the quiver $Q_{T}$ is a path and every path of length of two is a relation in the corresponding $m$-CTA.

Lemma 4.1. Suppose that $\mathcal{C}_{A_{n}}^{m}=D^{b}\left(K A_{n}\right) / F_{m}$, where $F_{m}=\tau^{-1}[m]$ with $m>$ $n-2$.

(1) $T_{1}, T_{2}, \ldots, T_{n}$ are $m$-diagonals of $\mathcal{P}_{m(n+1)+2}$.

(2) $T=T_{1} \oplus T_{2} \oplus \cdots \oplus T_{n}$ is an $m$-cluster tilting object.

(3) The $m$-cluster tilted algebra $\operatorname{End}^{o p}(T)$ is isomorphic to $K Q / \mathcal{I}$ where $Q$ is

$$
1 \stackrel{\alpha_{1}}{\longrightarrow} 2 \stackrel{\alpha_{2}}{\longrightarrow} 3 \rightarrow \cdots \rightarrow(n-1) \stackrel{\alpha_{n-1}}{\longrightarrow} n .
$$

and $\mathcal{I}$ is an ideal generated by all paths of length two. 
Proof. It is clear that $T_{1}, T_{2}, \ldots, T_{n}$ are $m$-diagonals of $\mathcal{P}_{m(n+1)+2}$, where if $i=n$ then $T_{n}=((n-(n-2)) m-(n-4),(n-(n-3)) m-(n-5))=(3 m-(n-5), 2 m-$ $(n-4))$. Observe that the picture of $m$-diagonals $T_{1}, T_{2}, \ldots, T_{n}$ in $\mathcal{P}_{m(n+1)+2}$ is Figure 14. Since $T_{1}, T_{2} \ldots, T_{n}$ are not crossing each other then $T$ is an $m$-cluster

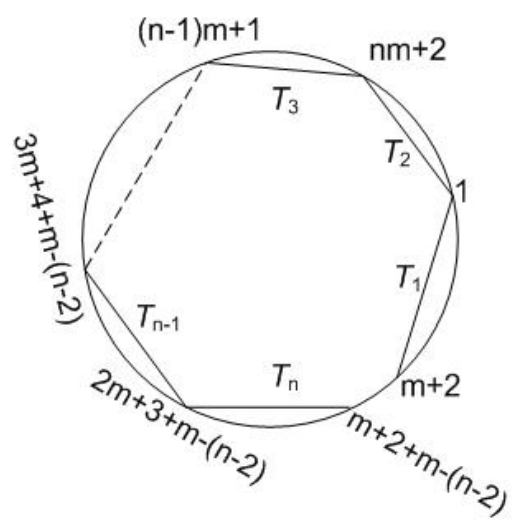

Figure 14. $m$-diagonals of $T$

tilting object. Let $Q$ be the quiver of $m$-cluster tilted algebra $\operatorname{End}^{o p}(T)$, then there exists exactly one arrow $T_{i} \rightarrow T_{i+1}$ for every $1 \leq i \leq n-1$. If $m>n-2$ then $m-(n-2)>0$ and consequently $m+2+m-(n-2)>m+2$. Hence, $T_{n}$ and $T_{1}$ don't have common endpoint. In other words there is no arrow from $T_{n}$ to $T_{1}$. We conclude $Q$ is the quiver in the proposition. Finally, by Lemma 2.5 the composition of all paths of length two is zero.

Lemma 4.2. Let $m \geq n-2$ and $T=T_{1} \oplus T_{2} \oplus \cdots \oplus T_{n-1} \oplus X_{1, i}$ with $1 \leq i \leq n-2$ then

(1) $T$ is an m-cluster tilting object in $\mathcal{C}_{A_{n}}^{m}$.

(2) If $Q$ is a quiver of $\operatorname{End}^{o p}(T)$ then $Q$ is

$$
1 \stackrel{\alpha_{1}}{\longrightarrow} 2 \stackrel{\alpha_{2}}{\longrightarrow} 3 \rightarrow \cdots \rightarrow n-1 \stackrel{\alpha_{n-1}}{\longrightarrow} n .
$$

(3) If $\rho_{j}=\alpha_{j} \alpha_{j+1}$ for every $1 \leq j \leq n-2$ then End ${ }^{o p}(T)=K Q / \mathcal{I}$ where $\mathcal{I}=\left\langle\rho_{1}, \rho_{2}, \ldots, \rho_{i-1}, \rho_{i+1}, \ldots, \rho_{n-2}\right\rangle$.

Proof. Suppose that $T^{\prime}=\left\{T_{1}, T_{2}, \ldots, T_{n-1}\right\}$ then it is clear that $T^{\prime}$ is the set of $m$-diagonals that are not crossing each other in $\mathcal{P}_{m(n+1)+2}$. We have that $X_{1,1}=$ $(1,2 m+2)$ and $X_{1, i}=(m(n-(i-2))-(i-4), 2 m-(i-3))$ for $1 \leq i \leq n-2=m$. Hence,

$$
m+2<2 m-(i-3)<2 m+3
$$

It follows that the set $T^{\prime} \cup\left\{X_{1, i}\right\}$ of $m$-diagonals in $\mathcal{P}_{m(n+1)+2}$ is as in Figure 15. We conclude that $T$ is an $m$-cluster tilting object of $C_{A_{n}}^{m}$. From Figure 15 we 


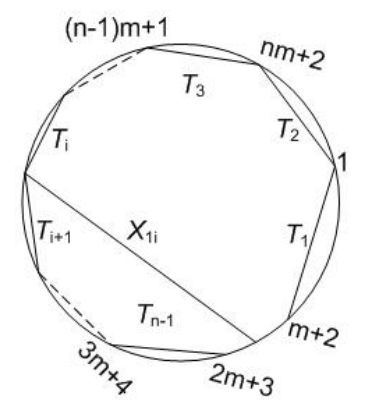

FiguRE 15. $m$-diagonal $T^{\prime} \cup X_{1, i}$

obtain easily that quiver of $\operatorname{End}^{o p}(T)$ is $Q$. Note that $m$-diagonals $T_{i}, X_{1, i}, T_{i+1}$ satisfy case 2 , hence the composition $\rho_{i}=\alpha_{i} \alpha_{i+1}$ is not zero. But all $\rho_{j}$ with $j \neq i$ is zero since the corresponding $m$-diagonals with $\rho_{j}$ satisfy case 1 . We conclude End $^{o p}(T) \cong K Q / \mathcal{I}$, as required.

Lemma above gives us how to construct other $m$-cluster tilting objects which have different relations. We know that the number of paths of length two in $A_{n}$ is $(n-2)$, where the relations are $\rho_{1}, \rho_{2}, \ldots, \rho_{n-2}$. In Lemma 4.2 ideal $\mathcal{I}$ is generated by a combination of $(n-3)$ relations of paths of length two from $(n-2)$ relations. We can get the $m$-CTA End $^{o p}(T) \cong K Q / \mathcal{I}$ where $\mathcal{I}$ generated by $(n-4)$ relations of paths of length two from $(n-2)$ relations by the following lemma.

Lemma 4.3. Suppose that $m \geq n-2$ and $T=T_{1} \oplus T_{2} \oplus \cdots \oplus T_{n-2} \oplus X_{1, i} \oplus X_{2, j}$ where $1 \leq i \leq j \leq n-3$ then $T$ is an $m$-cluster tilting object of $\mathcal{C}_{A_{n}}^{m}$. Furthermore, the algebra $\operatorname{End}^{o p}(T) \cong K Q / \mathcal{I}$ where $\mathcal{I}$ generated by $(n-4)$ relations of paths of length two. If $\mathfrak{T}$ be the collection of such $T$ then $|\mathfrak{T}|=\left(\begin{array}{l}n-2 \\ n-4\end{array}\right)$.

Proof. It is clear that $m$-diagonal $T_{1}, T_{2}, \ldots, T_{n-2}$ are not crossing each other in $\mathcal{P}_{m(n+1)+2}$. Now we just need to consider $m$-diagonals $X_{1, i}$ and $X_{2, j}$ in $\mathcal{P}_{m(n+1)+2}$. We have that

$$
\begin{aligned}
X_{1,1} & =(1,2 m+2), \\
X_{2,1} & =(1,3 m+2), \\
X_{1, i} & =(m(n-(i-2))-(i-4), 2 m-(i-3)) \text { and } \\
X_{2, j} & =(m(n-(j-2))-(j-4), 3 m-(j-3))
\end{aligned}
$$

where $i>1$ and $j>1$. It is easy to see that for $i=1$ and $j=1, m$-diagonals $T_{1}, T_{2}, \ldots, T_{n-2}, X_{1,1}, X_{2,1}$ are not crossing each other. Next, we consider endpoints of $X_{1, i}$ and $X_{2, j}$ for every $i \geq 1, j>1$. If $i=j$ then $3 m-(j-3)-(2 m-(i-3))=$ $m=n-2$. Since $j \leq n-3$ then

$$
m+2<m+4 \leq 2 m-(i-3)<3 m-(j-3) \leq 3 m+2<3 m+4 .
$$


It follows that one end point of $X_{1, i}$ and $X_{2, j}$ is in $\operatorname{arc} D_{m+2,3 m+4}$. While other point both of $X_{1, i}$ and $X_{2, j}$ coincides with one of endpoint of $T_{1}, T_{2}, \ldots, T_{n-2}$. It turns out that $X_{1, i}$ is not crossing with $T_{1}, T_{2}, \ldots, T_{n-2}$ as well as also for $X_{2, j}$. It remains to prove that $X_{1, i}$ and $X_{2, k}$ are not crossing each other. If $i=1$ and $j=1$ then it is clear that $X_{1,1}$ and $X_{2,1}$ are not crossing each other. If $i=1$ and $1<j \leq n-3$ then $X_{1,1}=(1,2 m+2)$ and $X_{2, j}=(m(n-(j-2))-(j-4), 3 m-(j-3))$ are not crossing each other. If $j \geq i>1$, we have $X_{1, i}=(m(n-(i-2))-(i-4), 2 m-(i-3))$ and $X_{2, j}=(m(n-(j-2))-(j-4), 3 m-(j-3))$. Since

$m(n-(j-2))-(j-4) \leq m(n-(i-2))-(i-4)$ and $2 m-(i-3)<3 m-(j-3)$

then $X_{1, i}$ and $X_{2, j}$ are not crossing each other. We deduce that $T_{1}, T_{2}, T_{n-2}, X_{1, i}, X_{2, j}$ is the set of $m$-diagonals which are not crossing each other. Thus, $T=T_{1} \oplus T_{2} \oplus$ $\cdots \oplus T_{n-2} \oplus X_{1, i} \oplus X_{2, j}$ is an $m$-cluster tilting object. Observe that paths of length two $X^{\prime} \rightarrow X_{1, i} \rightarrow X^{\prime \prime}$ and $Y^{\prime} \rightarrow X_{2, j} \rightarrow Y^{\prime \prime}$ with $X^{\prime}, Y^{\prime}, X^{\prime \prime}, Y^{\prime \prime}$ are $m$-diagonals of $T$ which satisfy case 2 in Lemma 2.1. Beside these two paths, all other path of length two in quiver $\operatorname{End}(T)$ satisfy case 1 in Lemma 2.1. Furthermore, for such $T$ there are exactly two paths of length two in $Q$ which composition in $\operatorname{End}^{o p}(T)$ is not zero .

We can compute the number of such $T$ by compute the number of all combinations $(i, j)$ where $1 \leq i \leq n-3$ and $i \leq j \leq n-3$.

TABLE 3. Pair of $(i, j)$

\begin{tabular}{|c|c|c|c|c|c|c|}
\hline$i$ & 1 & 2 & 3 & $\ldots$ & $n-2$ & $n-3$ \\
\hline$j$ & 1 & & & & & \\
& 2 & 2 & & & & \\
& 3 & 3 & 3 & & & \\
& $\vdots$ & $\vdots$ & $\vdots$ & $\vdots$ & $n-2$ & \\
& $n-3$ & $n-3$ & $n-3$ & $n-3$ & $n-3$ & $n-3$ \\
\hline
\end{tabular}

The number of such $T$ is

$$
1+2+\cdots+(n-4)+(n-3)=\frac{1}{2}(n-3)(n-2)=\frac{(n-2) !}{(n-4) ! 2 !} .
$$

We combine two lemmas above into a more general result, that is $m$-CTA End $^{o p}(T) \cong K Q / \mathcal{I}$ where $\mathcal{I}$ is an ideal generated by $(n-2-t)$ relations of paths of length two from $(n-2)$ relations and $1 \leq t \leq n-2$.

Lemma 4.4. Suppose that $m \geq n-2$ and $T=T_{1} \oplus T_{2} \oplus \cdots \oplus T_{n-t} \oplus X_{1, j_{1}} \oplus X_{2, j_{2}} \oplus$ $\cdots \oplus X_{t, j_{t}}$ with $1 \leq j_{1} \leq j_{2} \leq \cdots \leq j_{t} \leq n-t-1$ and $1 \leq t \leq n-2$, then $T$ is an $m$-cluster tilting object of $\mathcal{C}_{A_{n}}^{m}$. The $m$-cluster tilted algebra $\operatorname{End}^{\text {op }}(T) \cong k Q / \mathcal{I}$ where $\mathcal{I}$ is generated by $(n-2-t)$ relations of paths of length two. If $\mathfrak{T}$ be the collection of such $T$ then $|\mathfrak{T}|=\left(\begin{array}{c}n-2 \\ n-2-t\end{array}\right)$. 
Proof. For $t=1$ and $t=2$, it has been proved in Lemma 4.2 and Lemma 4.3. In general, we have that $m$-diagonals $T_{1}, T_{2}, \ldots, T_{n-t}$ are not crossing each other in regular gon $\mathcal{P}_{m(n+1)+2}$. Now consider $m$-diagonals $X_{1, j_{1}}, X_{2, j_{2}}, \ldots, X_{t, j_{t}}$ in $\mathcal{P}_{m(n+1)+2}$. If $m=n-2$ then

$T_{n-t}=((t+2) m-n+t+4,(t+3) m-n+t+5)=((t+1) m+t+2,(t+2) m+t+3)$.

We will see all cases of $j_{1}, j_{2}, \ldots, j_{t}$ in $\mathcal{P}_{m(n+1)+2}$. To show this we first consider the case $j_{1}=j_{2}=\cdots=j_{t}=1$ with the picture of this case in $\mathcal{P}_{m(n+1)+2}$ is

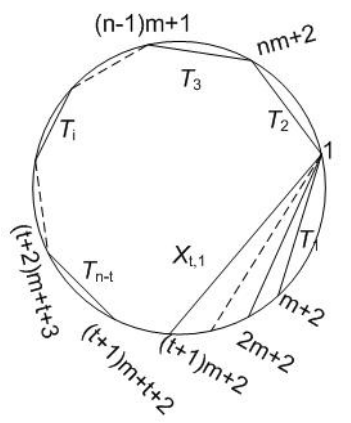

FiguRE 16. $m$-diagonals of $T$ in Lemma 4.4

We get that

$$
\begin{aligned}
X_{1, j_{1}} & =(1,2 m+2) \\
X_{2, j_{2}} & =(1,3 m+2) \\
\vdots & \\
X_{t-1, j_{t-1}} & =(1, t m+2) \\
X_{t, j_{t}} & =(1,(t+1) m+2) .
\end{aligned}
$$

The configuration of these $m$-diagonals in $\mathcal{P}_{m(n+1)+2}$ can be illustrated as in Figure 17. We will use that picture to see the other cases of $j_{1}, j_{2}, \ldots, j_{t}$. The upper line has $(n-t-1)$ black dots while the bottom line has $t$ black dots. Let us observe the $m$-diagonal $X_{i, j_{i}}=\left(x_{i}, y_{i}\right)$ where $x_{i}$ is one of the black dots on the upper line and $y_{i}$ one of the points (not necessarily black dot) on the bottom line. We have that $X_{k, 1}=(1,(k+1) m+2)$ with $1 \leq k \leq t$. We can conclude that $X_{i, j_{i}}=\left(x_{i}, y_{i}\right)$ where $x_{i}$ is the $j_{i}$-th black dot on the upper line counted from the right-hand side, and $y_{i}=(i+1) m+2-\left(j_{i}-1\right)=(i+1) m+3-j_{i}$. Suppose that $1 \leq i \leq t-1$ and $X_{i, j_{i}}=\left(x_{i}, y_{i}\right), X_{i+1, j_{i+1}}=\left(x_{i+1}, y_{i+1}\right)$ then

$$
y_{i}=(i+1) m+3-j_{i}<y_{i+1}=(i+1) m+3+m-j_{i+1} .
$$




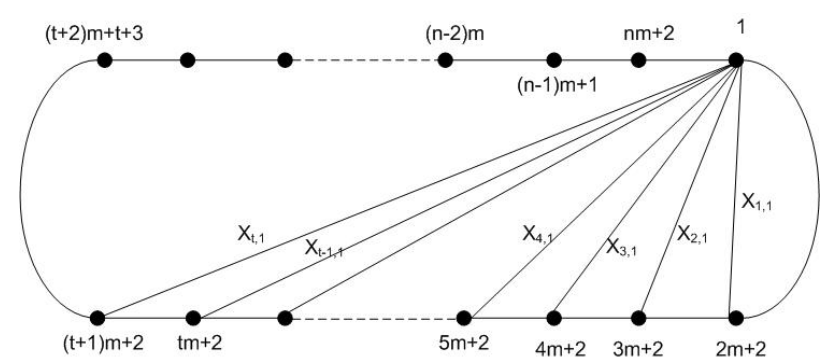

Figure 17. $m$-diagonals $X_{1,1}, X_{2,1}, \ldots, X_{t, 1}$

Since $j_{i} \leq j_{i+1} \leq n-t-1 \leq m$ then either $x_{i}=x_{i+1}$ or $x_{i+1}$ 's position is on the left of $x_{i}$. Moreover $i m+2<x_{i} \leq(i+1) m+2$. We describe this situation as in Figure 18 .

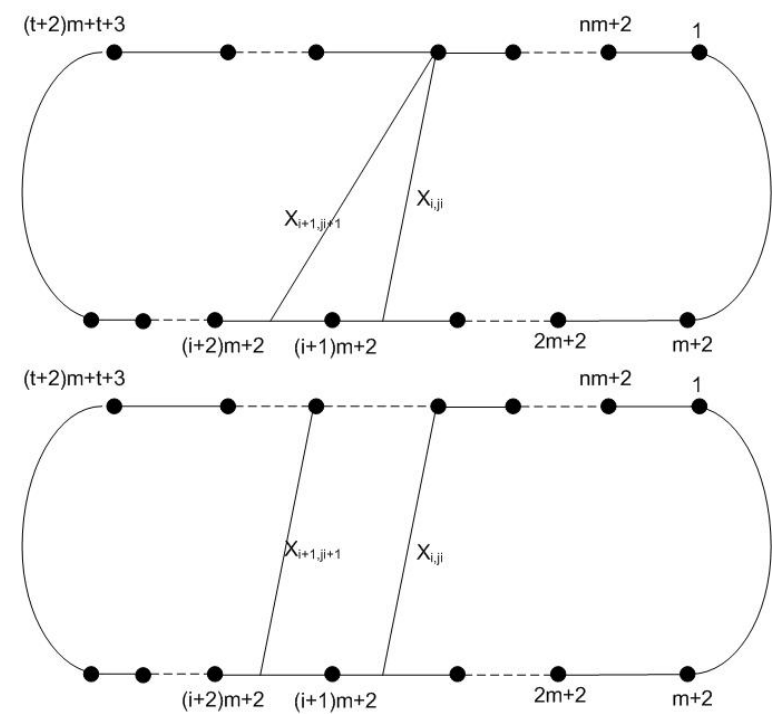

FIGURE 18. $m$-diagonals $X_{i, j_{i}}$ and $X_{i+1, j_{i+1}}$

Since $X_{i, j_{i}}=\left(x_{i}, y_{i}\right), X_{i+1, j_{i+1}}=\left(x_{i+1}, y_{i+1}\right)$ satisfy this condition(see Figure 18) for every $i$ then $X_{1, j_{1}}, X_{2, j_{2}}, \ldots, X_{t, j_{t}}$ are not crossing each other in $\mathcal{P}_{m(n+1)+2}$. Finally we conclude that $m$-diagonals $T_{1}, T_{2}, \ldots, T_{n-t}, X_{1, j_{1}}, X_{2, j_{2}}, \ldots, X_{t, j_{t}}$ are not crossing each other in regular gon $\mathcal{P}_{m(n+1)+2}$, it proves that $T$ is an $m$-cluster tilting object. Next we show the last statement. Every $m$-diagonal $X_{i, j_{i}}$ represent one path of length two which is not zero in $\operatorname{End}^{o p}(T)$. Hence, there exists $(n-2-t)$ relations of paths of length two in $\operatorname{End}^{o p}(T)$. Now we compute the number of 
$T$ in this theorem. This number equal to the number of possibilities of $t$-tuple $\left(j_{1}, j_{2}, \ldots, j_{t}\right)$ where $1 \leq j_{1} \leq j_{2} \leq \cdots \leq j_{t} \leq n-t-1$. This problem is equivalent to counting the number of distinct shortest routes from point A to point B in the the following diagram :

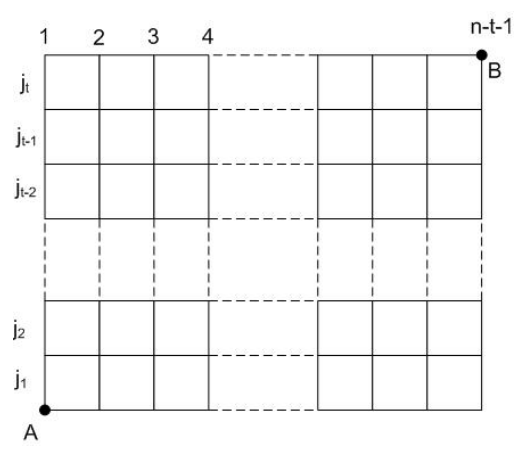

Figure 19. Map of routes from $A$ to $B$

Here $j_{i}$ interpreted as a step up to the $i$-th and for every $j_{i}$ there is $(n-t-1)$ positions can be chosen. It is easy to see that the number of distinct shortest route is combination $(n-2-t)$ from $(n-2)$, that is

$$
\left(\begin{array}{c}
n-2 \\
n-2-t
\end{array}\right)=\frac{(n-2) !}{t !(n-2-t) !}
$$

Proposition 4.5. Let $m=n-2$ and $H=K Q / \mathcal{I}$ where $Q$ is quiver

$$
1 \stackrel{\alpha_{1}}{\longrightarrow} 2 \stackrel{\alpha_{2}}{\longrightarrow} 3 \rightarrow \cdots \rightarrow n-1 \stackrel{\alpha_{n-1}}{\longrightarrow} n .
$$

Let $W=\left\{\rho_{j}=\alpha_{j} \alpha_{j+1} \mid 1 \leq j \leq n-2\right\}$ and $B \subseteq W,|B| \neq n-2$. If $\mathcal{I}=\langle B\rangle$ then $H$ is an $m-C T A$.

Proof. If $B=\emptyset$ then $I=0$, we choose $T$ in Lemma 4.4 with $t=n-2$ hence we get $\operatorname{End}^{o p}(T)=K Q$. If $|B|=k>1$, by Lemma 4.4 we can choose $T$ with $t=n-2-k$ such that $\operatorname{End}^{o p}(T) \cong H$.

So far we have obtain some $m$-CTAs in case $m=n-2$. By Theorem 3.1 it remains to find $m$-CTAs whose number of relations is $n-2$. But we will show that there is no such $m$-CTA.

Lemma 4.6. If $m=n-2$ then there is no $m$-cluster tilting object $T$ of $\mathcal{C}_{A_{n}}^{m}$ such that $\operatorname{End}^{o p}(T) \cong K Q / \mathcal{I}$ with $\mathcal{I}=\left\langle\rho_{1}, \rho_{2}, \ldots, \rho_{n-1}, \rho_{n-2}\right\rangle$. 


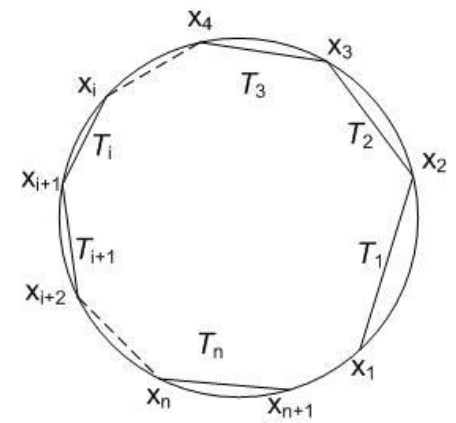

FIGURE 20. $m$-diagonals $T_{1}, T_{2}, \ldots, T_{n}$

Proof. Let $T_{1}, T_{2}, \ldots, T_{n}$ be $m$-diagonals corresponding to $T$, then by Lemma 2.5 , these $m$ - diagonal in $\mathcal{P}_{m(n+1)+2}$ should be as in Figure 20. It means that $x_{n+1} \neq x_{1}$ or equivalently arc $D_{x_{1} x_{n+1}}$ has at least one side. Note that $\operatorname{arc} D_{x_{i+1} x_{i}}$ has at least $m+1$ side. If all $T_{i}$ are short then without loss of generality, suppose that $x_{1}=m+2$ and $x_{2}=1$. Consequently, $T_{1}=(1, m+2), T_{2}=(1, n m+2), T_{3}=$ $((n-1) m+1, n m+2), T_{4}=((n-1) m+1,(n-2) m)$ and for $5 \leq i \leq n$,

$$
T_{i}=((n-(i-2)) m-(i-4),(n-(i-3)) m-(i-5)) .
$$

The number of sides in $\operatorname{arc} D_{x_{n+1} x_{1}}$ is $(m+1) n=m n+n$. Hence, the number of sides in $\operatorname{arc} D_{x_{1} x_{n+1}}$ is

$$
m(n+1)+2-(m n+n)=m-(n-2) .
$$

However if $m=n-2$ then there is no side in arc $D_{x_{1} x_{n+1}}$, a contradiction. Now suppose that there exists $T_{j}$ which is not short. It follows that the number of sides in $\operatorname{arc} D_{x_{n+1} x_{1}}$ is more than $(m+1) n$. If $x$ is the number of sides in $\operatorname{arc} D_{x_{n+1} x_{1}}$ then $x>m n+n$. We have that $(m(n+1)+2-x)$ is the number of side in arc $D_{x_{1} x_{n+1}}$. Consequently

$$
m(n+1)+2-x<m(n+1)+2-(m n+n)=m-(n-2)=0
$$

since $m=n-2$, a contradiction. We conclude that there is no such $T$.

We end this section by giving all $m$-CTAs which are Nakayama algebras with acyclic quiver in the case $m \geq n-2$.

Proposition 4.7. Let $m=n-2$ and $H \cong K Q / \mathcal{I}$ be an algebra with $Q$ is

$$
1 \stackrel{\alpha_{1}}{\longrightarrow} 2 \stackrel{\alpha_{2}}{\longrightarrow} 3 \rightarrow \cdots \rightarrow n-1 \stackrel{\alpha_{n-1}}{\longrightarrow} n .
$$

The algebra $H$ is an $m-C T A$ of $\mathcal{C}_{A_{n}}^{m}$ if only if $\mathcal{I}$ is generated by at most $(n-3)$ paths of length two.

Proof. Use Theorem 2.6, Corollary 4.5 and Lemma 4.6. 
Proposition 4.8. Let $m>n-2$ and $H=K Q / \mathcal{I}$ with $Q$ is the quiver

$$
1 \stackrel{\alpha_{1}}{\longrightarrow} 2 \stackrel{\alpha_{2}}{\longrightarrow} 3 \rightarrow \cdots \rightarrow n-1 \stackrel{\alpha_{n-1}}{\longrightarrow} n .
$$

Suppose that $W=\left\{\rho_{j}=\alpha_{j} \alpha_{j+1} \mid 1 \leq j \leq n-2\right\}$ and $B \subseteq W$. If $\mathcal{I}=\langle B\rangle$ then $H$ is an $m-C T A$.

Proof. If $B \neq W$, we choose $m$-cluster tilting object $T$ in Lemma 4.4. If $B=W$ then we choose the $m$-cluster tilting object $T$ in Lemma 4.1.

Theorem 4.9. Let $m>n-2$ and $H \cong K Q / \mathcal{I}$ be an algebra with $Q$ is the quiver

$$
1 \stackrel{\alpha_{1}}{\longrightarrow} 2 \stackrel{\alpha_{2}}{\longrightarrow} 3 \rightarrow \cdots \rightarrow(n-1) \stackrel{\alpha_{n-1}}{\longrightarrow} n .
$$

The algebra $H$ is an m-CTA of $\mathcal{C}_{A_{n}}^{m}$ if only if $\mathcal{I}$ is generated by any collection of paths of length two.

Proof. Apply Theorem 2.6 and Proposition 4.8.

\subsection{Case $m<n-2$.}

Just like in the two previous cases to characterize Nakayama $m$-CTA, in this case it is sufficient to simply consider the relations of path of length two that appear on this algebra. If the number of relations is at most $m$, then there is $m$-cluster tilting object such that the corresponding $m$-CTA is Nakayama algebra. If the ideal generated by more than $m$ relations of paths of length two we have not been able to guarantee which algebras are Nakayama $m$-CTA. This happens because we get different cases depending on the difference between $m$ and $n-2$ (we denote by a). In the first part we put forward some Nakayama algebra which are not $m$-CTA in the case $m<n-2$. This class of algebra are given in Lemma 4.10, Lemma 4.11, Lemma 4.12 and Lemma 4.13. Next, we provide all the Nakayama $m$-CTA algebras which have at most $m$ relation of path of length two in Lemma 4.14 parts (ii), (iii) and Lemma 4.16 parts (ii). In Theorem 4.18 we give a characterization of Nakayama $m$-CTA which have at most $m$ relations. In the last part we try to find the possibility of more than $m$ relations of path of length two. In Proposition 4.19 there are Nakayama algebras with more than $m$ relation which are not $m$-CTA for some certain condition of $a$. We also give Nakayama algebras with more than $m$ relation which are $m$-CTA for some certain condition in Proposition 4.20.

We begin by giving Nakayama algebras acyclic type which are not $m$-CTAs.

Lemma 4.10. If $m<n-2$ then there is no $m$-cluster tilting object $T$ in $\mathcal{C}_{A_{n}}^{m}$ such that $\operatorname{End}^{o p}(T) \cong K Q / \mathcal{I}$ with $Q$ is

$$
1 \stackrel{\alpha_{1}}{\longrightarrow} 2 \stackrel{\alpha_{2}}{\longrightarrow} 3 \rightarrow \cdots \rightarrow(n-1) \stackrel{\alpha_{n-1}}{\longrightarrow} n
$$

and $\mathcal{I}=\left\langle\rho_{1}, \rho_{2}, \ldots, \rho_{n-3}, \rho_{n-2}\right\rangle$, where $\rho_{i}=\alpha_{i} \alpha_{i+1}$ for every $i$.

Proof. We utilize the same methods as in the proof of Lemma 4.6. If $T_{1}, T_{2}, \ldots, T_{n}$ are $m$-diagonals correspond to $T$ then by Lemma 2.5 , these $n m$-diagonals in $\mathcal{P}_{m(n+1)+2}$ should be as Figure 21, and it turns out that $\operatorname{arc} D_{x_{1} x_{n+1}}$ at least 


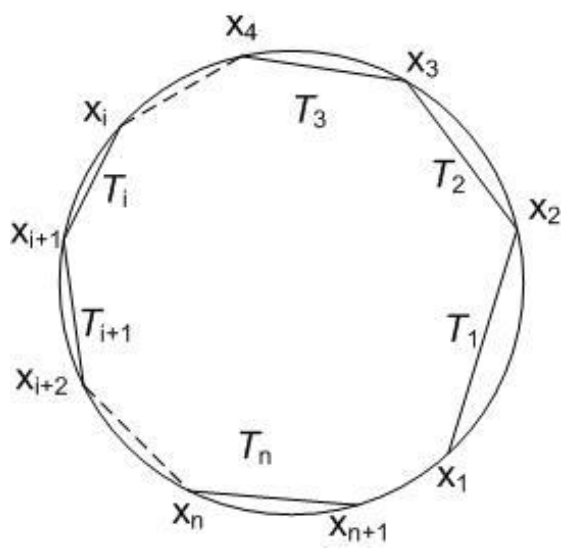

Figure 21. $m$-diagonals $T_{1}, T_{2}, \ldots, T_{n}$

has one side. Note that the number of sides in $\operatorname{arc} D_{x_{i+1} x_{i}}$ is at least $m+1$. Therefore, $\operatorname{arc} D_{x_{n+1} x_{1}}$ has at least $(m n+n)$ sides. Let $x$ be the number of sides in arc $D_{x_{n+1} x_{1}}$, then $x \geq m n+n$. We also have that $(m(n+1)+2-x)$ is the number of sides in $\operatorname{arc} D_{x_{1} x_{n+1}}$. Therefore

$$
m(n+1)+2-x \leq m(n+1)+2-m n-n=m-(n-2)<0,
$$

because $m<n-2$, a contradiction. The proof is complete.

Next lemma shows that the Nakayama algebra whose relations are $m+1$ consecutive relation paths of length two starting from $\rho_{a+1}$ is not $m$-CTA.

Lemma 4.11. Suppose that $m<n-2$ and $a=n-2-m$ then there is no $m$-cluster tilting object $T$ of $\mathcal{C}_{A_{n}}^{m}$ such that $\operatorname{End}^{o p}(T) \cong K Q / \mathcal{I}$ with $Q$ is

$$
1 \stackrel{\alpha_{1}}{\longrightarrow} 2 \stackrel{\alpha_{2}}{\longrightarrow} 3 \rightarrow \cdots \rightarrow(n-1) \stackrel{\alpha_{n-1}}{\longrightarrow} n
$$

and $\mathcal{I}=\left\langle\rho_{a+1}, \rho_{a+2}, \ldots, \rho_{n-3}, \rho_{n-2}\right\rangle$, where $\rho_{i}=\alpha_{i} \alpha_{i+1}$ for every $i$.

Proof. Suppose that there exists such T. By Lemma 2.1, the configuration of $m$ diagonals correspond to $T$ in $\mathcal{P}_{m(n+1)+2}$ is as in Figure 22. Hence we may write $T=Y_{1} \oplus Y_{2} \oplus \cdots \oplus Y_{m+2} \oplus X_{1} \oplus X_{2} \oplus \cdots \oplus X_{a}$. It follows that arc $D_{x_{a+1} y_{m+2}}$ has at least one side. By the definition of $m$-diagonal, arc $D_{y_{i+1} y_{i}}$ and arc $D_{x_{1} y_{1}}$ have at least $m+1$ sides, while arc $D_{x_{j} x_{j+1}}$ has at least $m$ side. Hence, arc $D_{y_{m+2} x_{a+1}}$ has at least

$$
(m+2)(m+1)+a m=(m+2)(m+1)+(n-2-m) m=m(n+1)+2
$$

sides. A contradiction since $\mathcal{P}_{m(n+1)+2}$ has $m(n+1)+2$ sides and arc $D_{x_{a+1} y_{m+2}}$ has at least one side. 


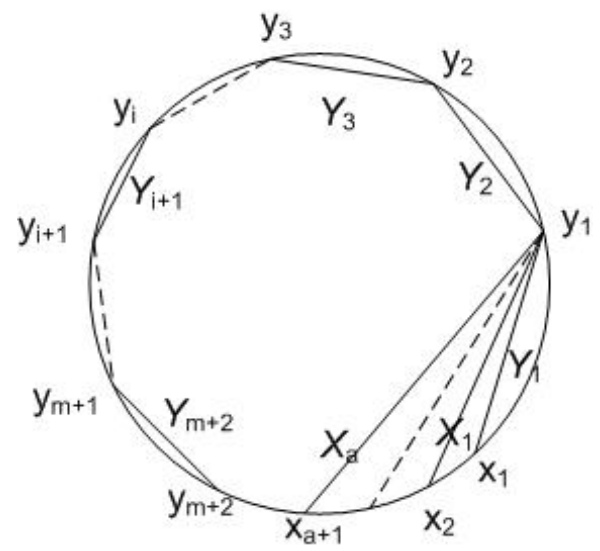

Figure 22. $m$-diagonals $Y_{1}, Y_{2}, \ldots, Y_{m+2}, X_{1}, X_{2}, \ldots, X_{a}$

We have that Nakayama algebra with $m$ consecutive relations of path of length two is not $m$-CTA of type $A_{n}$.

Lemma 4.12. Suppose that $m<n-2$ and $a=n-2-m$ then there is no $m$-cluster tilting object $T$ of $\mathcal{C}_{A_{n}}^{m}$ such that $\operatorname{End}^{o p}(T) \cong K Q / \mathcal{I}$ with $Q$ is

$$
1 \stackrel{\alpha_{1}}{\longrightarrow} 2 \stackrel{\alpha_{2}}{\longrightarrow} 3 \rightarrow \cdots \rightarrow(n-1) \stackrel{\alpha_{n-1}}{\longrightarrow} n
$$

and $\mathcal{I}=\left\langle\rho_{t}, \rho_{t+1}, \ldots, \rho_{t+m-1}\right\rangle$ where $1 \leq t \leq a$, where $\rho_{i}=\alpha_{i} \alpha_{i+1}$ for every $i$.

Proof. Assume that there exists such $T$, then we have $m$ paths of length two in $\operatorname{End}^{o p}(T)$ whose composition is zero. Therefore we need exactly $m$ triplets of $m$ diagonals satisfy case 1 in Lema 2.1. Since the quiver of $\operatorname{End}^{o p}(T)$ is a path then there exist $(m+2) m$-diagonals in $\mathcal{P}_{m(n+1)+2}$, where the configuration is as in Figure 23. Thus it remains a $m$-diagonals. Because $\mathcal{I}=\left\langle\rho_{t}, \rho_{t+1}, \ldots, \rho_{t+m-1}\right\rangle$ then we should have $(t-1) m$-diagonals whose endpoint is $y_{1}$ and the other endpoint in arc $D_{x_{1} x_{m+2}}$ while the remaining $(a-(t-1)) m$-diagonals have one endpoint at $y_{m+1}$ and the other point in arc $D_{x_{1} y_{m+2}}$. More precisely, the picture of all $m$-diagonals should be like Figure 24. From Figure 24, $m$-diagonals which correspond to $T$ are $T_{1}, T_{2}, \ldots, T_{m+2}, X_{1}, X_{2}, \ldots, X_{t-1}$,

$Y_{1}, Y_{2}, \ldots, Y_{a-t+1}$ with $X_{i}=\left(y_{1}, x_{i+1}\right)$ and $Y_{j}=\left(y_{m+1}, z_{j}\right)$. Note that for every $1 \leq i \leq t-1, \operatorname{arc} D_{x_{i} x_{i+1}}$ has at least $m$ sides. We also have that either arc $D_{x_{j} x_{j-1}}$ or $\operatorname{arc} D_{z_{1} y_{m+1}}$ has at least $m$ sides. Hence, the number of sides in $\operatorname{arc} D_{z_{a-t+1} x_{t}}$ is at least

$(m+1)(m+2)+(t-1) m+(a-t+1) m=(m+1)(m+2)+a m=m(n+1)+2$, this contradicts the fact that arc $D_{x_{t} z_{a-t+1}}$ has at least one side.

The following lemma states that Nakayama algebra with consecutive relations of path of length two ending in $\rho_{n-2}$ is not $m$-CTA of type $A_{n}$. 


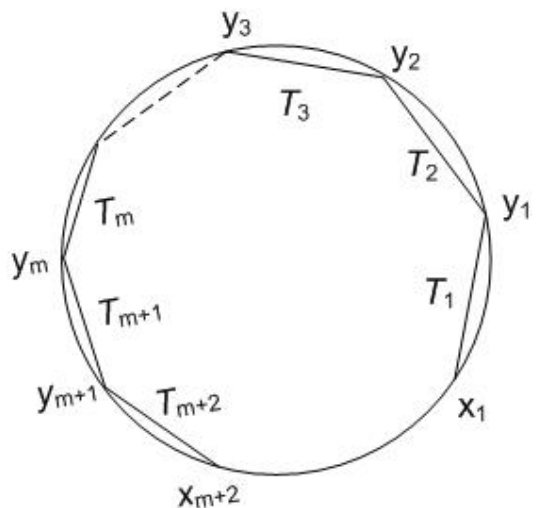

Figure 23. $m$-diagonals $T_{1}, T_{2}, \ldots, T_{m+2}$

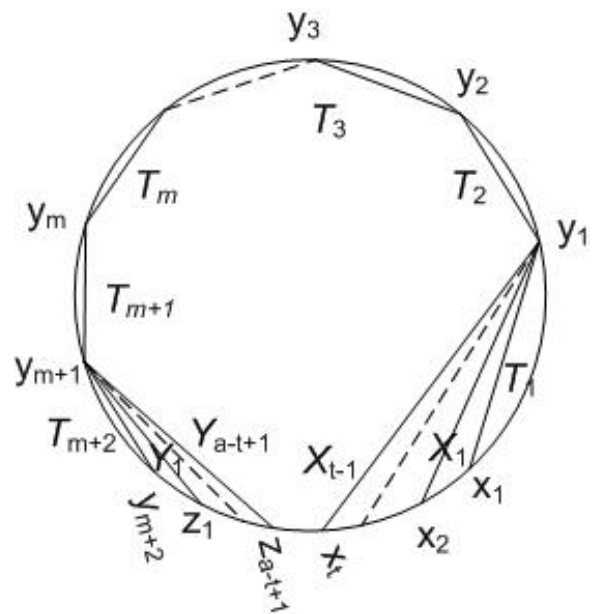

Figure 24. $m$-diagonals $T_{1}, \ldots, T_{m+2}, X_{1}, X_{2}, \ldots, X_{t-1}, Y_{1}, \ldots, Y_{a-t+1}$

Lemma 4.13. Suppose that $m<n-2$ and $a=n-2-m$ then there is no $m$-cluster tilting object $T$ of $\mathcal{C}_{A_{n}}^{m}$ such that $\operatorname{End}^{\text {op }}(T) \cong K Q / \mathcal{I}$ with $Q$ is

$$
1 \stackrel{\alpha_{1}}{\longrightarrow} 2 \stackrel{\alpha_{2}}{\longrightarrow} 3 \rightarrow \cdots \rightarrow(n-1) \stackrel{\alpha_{n-1}}{\longrightarrow} n
$$

and $\mathcal{I}=\left\langle\rho_{j+1}, \rho_{j+2}, \ldots, \rho_{n-3}, \rho_{n-2}\right\rangle$ for every $0 \leq j \leq a$, where $\rho_{i}=\alpha_{i} \alpha_{i+1}$ for every $i$.

Proof. The cases $j=0$ and $j=a$ have been proved in Lemma 4.10 and Lemma 4.11. Now assume that $1<j<a$, then the picture of $m$-diagonals which corresponds to 
$T$ in $\mathcal{P}_{m(n+1)+2}$ is Observe that arc $D_{y_{n-j} x_{1}}$ has at least $(m+1)(n-j)$ sides, while

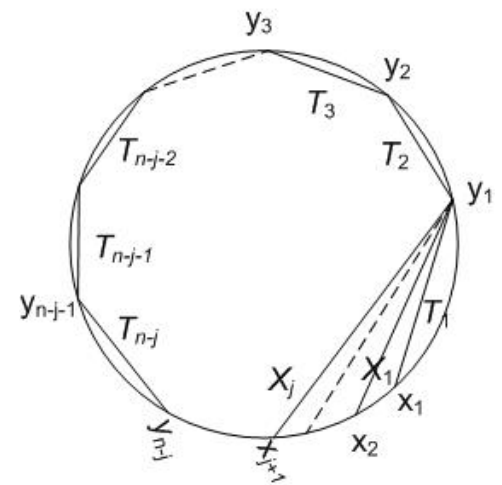

Figure 25. $m$-diagonals $T_{1}, T_{2}, \ldots, T_{n-j}, X_{1}, \ldots, X_{j}$

$\operatorname{arc} D_{x_{1} x_{j+1}}$ has at least $j m$ sides. Thus, the number of sides in $\operatorname{arc} D_{y_{n-j} x_{j+1}}$ is at least

$$
(m+1)(n-j)+j m=m n-j m+n-j+j m=n(m+1)-j .
$$

Since $j<a$ we have

$$
m(n+1)-j>m(n+1)-a=n(m+1)-(n-2-m)=m(n+1)+2 .
$$

This contradicts the fact that $\mathcal{P}_{m(n+1)+2}$ has $(m(n+1)+2)$ sides.

Lemma 4.14. Suppose that $m<n-2, a=n-2-m$ and $T=T_{1} \oplus T_{2} \oplus \cdots \oplus$ $T_{n-t} \oplus X_{1, j_{1}} \oplus X_{2, j_{2}} \oplus \cdots \oplus X_{t, j_{t}}$ with $1 \leq j_{1} \leq j_{2} \leq \cdots \leq j_{t} \leq \min \{m, n-t-1\}$ and $a \leq t \leq n-2$ then $T$ is an m-cluster tilting object of $\mathcal{C}_{A_{n}}^{m}$.

(i) If $t=a$ and $j_{t}=1$ then the algebra $\operatorname{End} d^{o p}(T) \cong K Q / \mathcal{I}$ with $Q$ is

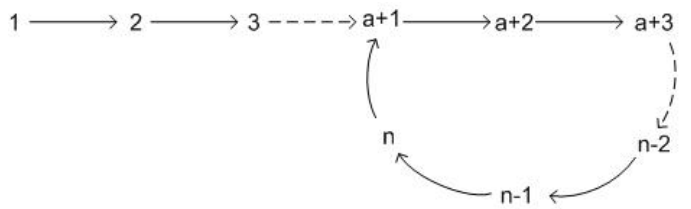

and $\mathcal{I}$ generated by all paths of length two in the cycle.

(ii) If $t>$ a then $\operatorname{End}^{o p}(T) \cong K Q / \mathcal{I}$ with $Q$ is

$$
1 \stackrel{\alpha_{1}}{\longrightarrow} 2 \stackrel{\alpha_{2}}{\longrightarrow} 3 \rightarrow \cdots \rightarrow(n-1) \stackrel{\alpha_{n-1}}{\longrightarrow} n
$$

and $\mathcal{I}$ generated by $(n-2-t)$ relations of paths of length two from $(n-2)$ relations of paths of length two.

(iii) If $t=a$ and $j_{t} \neq 1$ then $\operatorname{End}^{o p}(T) \cong K Q / \mathcal{I}$ with $Q$ is the quiver in part (ii) and $\mathcal{I}$ generated by $m$ relations of paths of length two where $\rho_{n-2} \in \mathcal{I}$. 
Proof. First, consider case $t=a$ and $j_{t}=1$, we get $j_{1}=j_{2}=\cdots=j_{t}=1$. Consequently $T=T_{1} \oplus T_{2} \oplus \cdots \oplus T_{m+2} \oplus X_{1,1} \oplus X_{2,1} \oplus \cdots \oplus X_{a, 1}$. We have that $T_{m+2}=(m(a+1)+2, m(a+2)+3)$ and $X_{a, j_{a}}=X_{a, 1}=(1, m(a+1)+2)$, it follows that $T_{m+2}$ and $X_{a, 1}$ have a common endpoint $m(a+1)+2$. Hence, the picture of $m$-diagonals that corresponds to $T$ is as in Figure 26. It is clear

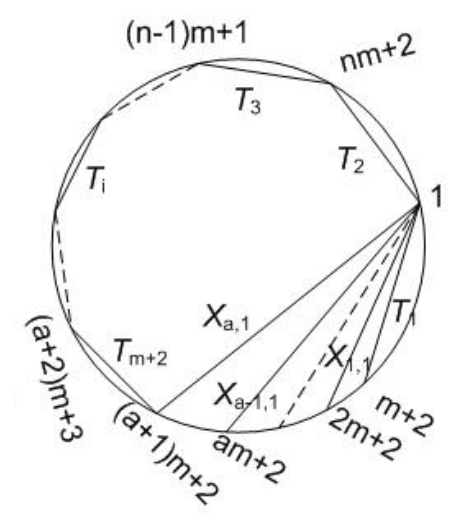

Figure $26 . \quad m$-diagonals $T_{1}, T_{2}, \ldots, T_{m+1}, X_{1,1}, \ldots, X_{a, 1}$

that the algebra $\operatorname{End}^{o p}(T)$ satisfies the first part of the lemma. Furthermore, if $t>a$ then $(t+1) m+2+t+m-n-2>(t+1) m+2$. Therefore $T_{n-t}=$ $((t+1) m+2+t+m-n-2,(t+2) m+3+t+m-n-2)$ and $X_{t, 1}=(1,(t+1) m+2)$ either are not crossing each other or have a common endpoint in $\mathcal{P}_{m(n+1)+2}$. Since $t \geq a+1$ then $\min \{m, n-t-1\}=m+1$ or $\min \{m, n-t-1\}=n-t-1$. It follows that

$$
1 \leq j_{1} \leq j_{2} \leq \cdots \leq j_{t} \leq \min \{m, n-t-1\} \leq m
$$

and then we may use the same way as in the proof of Lemma 4.4. If $t=a$ then $T=T_{1} \oplus T_{2} \oplus \cdots \oplus T_{m+2} \oplus X_{1, j_{1}} \oplus X_{2, j_{2}} \oplus \cdots \oplus X_{a, j_{a}}$. The fact that $m$-diagonals which correspond to $T$ are not crossing each other can be obtained by the same argument as in the proof of Lemma 4.4. Because $2 \leq j_{a} \leq m$ we have that $X_{a, j_{a}}$ does not have a common endpoint neither with $T_{m+2}$ nor at the point $a m+2$. Thus, we have the quiver of $\operatorname{End}^{o p}(T)$ is $A_{n}$. Next, we will prove that $\rho_{n-2} \in \mathcal{I}$. Consider $m$-diagonals $T_{m}, T_{m+1}$ and $T_{m+2}$ in $\mathcal{P}_{m(n+1)+2}$ in Figure 27 .

Since $j_{i} \leq m$ then there is no $m$-diagonal $X_{i, j_{i}}$ that have a common endpoint at $y_{m+1}$. So there exists an irreducible map $T_{m} \rightarrow T_{m+1} \rightarrow T_{m+2}$. Because at the point $x_{m+2}$ there is only one $m$-diagonal $T_{m+2}$ then this irreducible map corresponds to the path $\alpha_{n-2} \alpha_{n-1}$ in $Q$. But this path satisfies case 1 in Lemma 2.1, hence by Lemma 2.5, $\rho_{n-2}=0$ in $\operatorname{End}^{o p}(T)$. 


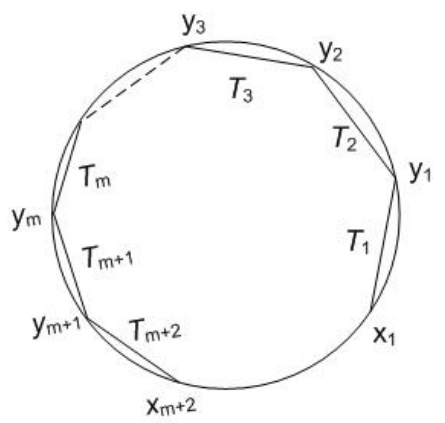

Figure 27. $m$-diagonals $T_{1}, T_{2}, \ldots, T_{m+2}$
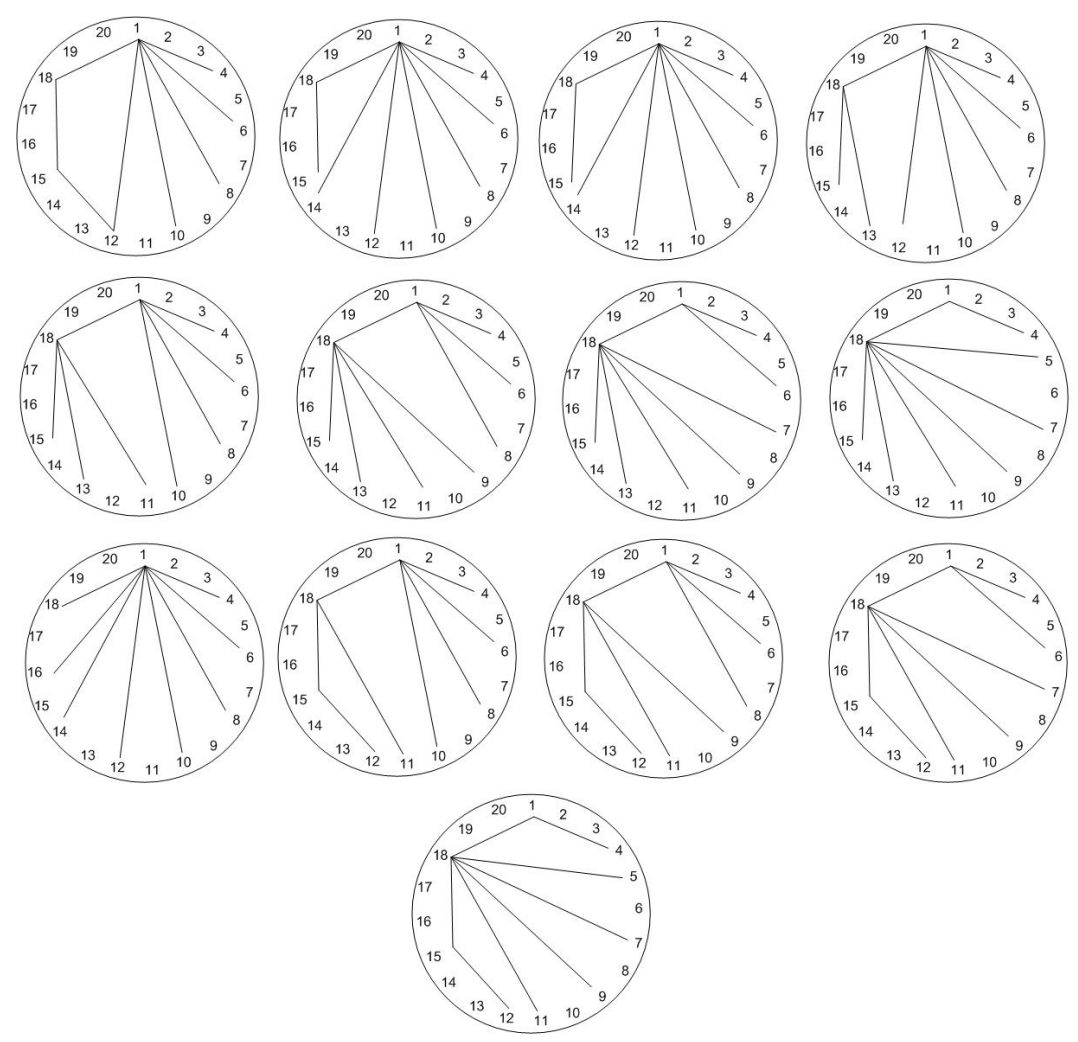

FiguRE 28. $m$-diagonals of $T$ for $m=2$ and $n=8$

Example 4.15. Let $m=2$ and $n=8$ then $a=8-2-2=4$ and $m(n+1)+2=20$. All $m$-diagonals which correspond to $T$ in Lemma 4.14 are as in Figure 28 
Lemma 4.16. Suppose that $m<n-2, a=n-2-m$ and $T=T_{1} \oplus \cdots \oplus T_{m+2} \oplus X_{1, j_{1}} \oplus X_{2, j_{2}} \oplus \cdots \oplus X_{k, j_{k}} \oplus X_{k+1, m+1} \oplus \cdots \oplus X_{a-1, m+1} \oplus X_{a, m+1}$ with $1 \leq j_{1} \leq j_{2} \cdots \leq j_{k} \leq m$ and $1 \leq k<a$ then $T$ is an $m$-cluster tilting object of $\mathcal{C}_{A_{n}}^{m}$.

(i) If $j_{k}=1$ then the algebra $\operatorname{End}^{o p}(T) \cong K Q / \mathcal{I}$ with $Q$ is and $\mathcal{I}$ generated by

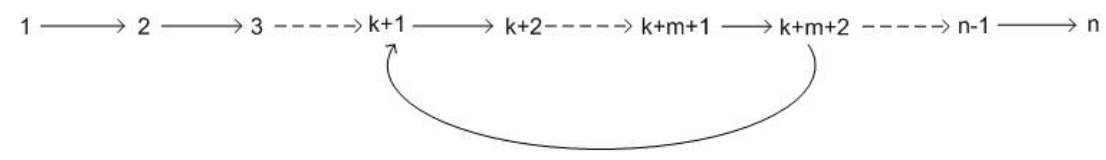

all paths of length two in the cycle.

(ii) If $j_{k} \neq 1$ and $j_{k} \leq m$ then the algebra $\operatorname{End} d^{o p}(T) \cong K Q / \mathcal{I}$ with $Q$ is

$$
1 \stackrel{\alpha_{1}}{\longrightarrow} 2 \stackrel{\alpha_{2}}{\longrightarrow} 3 \rightarrow \cdots \rightarrow(n-1) \stackrel{\alpha_{n-1}}{\longrightarrow} n
$$

and $\mathcal{I}$ generated by $m$ relations of paths of length two with $\rho_{k+m+1}, \rho_{k+m+2}, \ldots, \rho_{n-3}, \rho_{n-2} \notin \mathcal{I}$ and $\rho_{k+m} \in \mathcal{I}$.

Proof. Note that for $k=a, T$ is the $m$-cluster tilting object in Lemma 4.14 part 1. Assume that $k<a$, if $j_{k}=1$ then $T=T_{1} \oplus \cdots \oplus T_{m+2} \oplus X_{1,1} \oplus X_{2,1} \oplus$ $\cdots \oplus X_{k, 1} \oplus X_{k+1, m+1} \oplus \cdots \oplus X_{a-1, m+1} \oplus X_{a, m+1}$. We have that $m$-diagonal $X_{k, 1}=(1,(k+1) m+2)$ and $X_{k+1, m+1}=X_{k+1,2}$ if $m=1$ or

$$
\begin{aligned}
X_{k+1, m+1} & =((n-(m+1-2)) m-(m+1-4),(k+1+1) m-(m+1-3)) \\
& =((n-m) m+3,(k+1) m+2)
\end{aligned}
$$

if $m \neq 1$. If $m \neq 1$ then $X_{k, 1}$ and $X_{k+1, m+1}$ have a common endpoint at $(k+1) m+2$. If $m=1$ then $k=1$ and hence $X_{k+1, m+1}=X_{2,2}=(n+2,4), X_{k, 1}=X_{1,1}=$ $(1,4)$. It turns out that $X_{k+1, m+1}$ and $X_{k, 1}$ have a common endpoint if $m=$ 1. So the picture of $m$-diagonals which correspond to $T=T_{1} \oplus \cdots \oplus T_{m+2} \oplus$ $X_{1,1} \oplus X_{2,1} \oplus \cdots \oplus X_{k, 1} \oplus X_{k+1, m+1} \oplus \cdots \oplus X_{a-1, m+1} \oplus X_{a, m+1}$ in $\mathcal{P}_{m(n+1)+2}$ is as in Figure 29. For $j_{k} \neq 1$ the configuration of $m$-diagonals $T_{1}, T_{2}, \ldots, T_{m+2}$ and $X_{k+1, m+1}, \ldots, X_{a-1, m+1}, X_{a-1, m+1}$ in $\mathcal{P}_{m(n+1)+2}$ is the same as in the Figure 29. It remains to consider the position of $X_{1, j_{1}}, X_{2, j_{2}}, \ldots, X_{k, j_{k}}$ in $\mathcal{P}_{m(n+1)+2}$ if $j_{k} \neq 1$ and $j_{k} \leq m$. By the same arguments as in the proof of Lemma 4.4 then for $X_{i, j_{i}}$ and $X_{i+1, j_{i+1}}$ in $\mathcal{P}_{m(n+1)+2}$ will be one of the following pictures in Figure 30. If $j_{k} \leq m$ then the number of black dots on the top line that can be the end point of $X_{i, j_{i}}$ except point 1 is $m$ (see Figure 30). Consequently the leftmost black dot on the top line is $(a+3) m+4$. We claim that the ideal $\mathcal{I}$ generated by $m$ relations of paths of length two. From Figure 29 we have that $T_{2}, T_{3}, \ldots, T_{m+1}$ are $m$-diagonals that correspond to a midpoint of a path of length two that satisfies case 1 in Lemma 2.1 while others $m$-diagonal satisfy case 2 in Lemma 2.1 . So the number of relations that generate $\mathcal{I}$ is only $m$. 


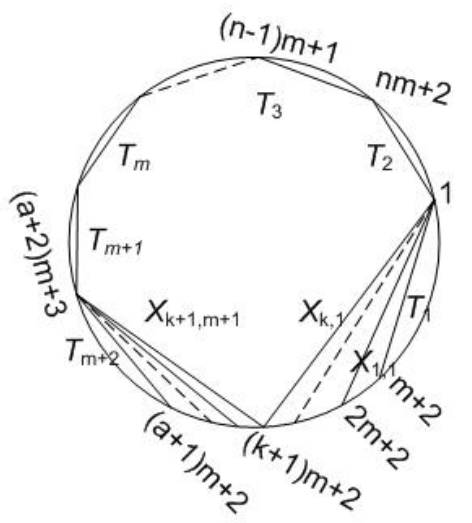

Figure 29. $m$-diagonals $T_{1}, \ldots, T_{m+2}, X_{1,1}, X_{2,1}, \ldots, X_{k, 1}, X_{k+1, m+1}, \ldots, X_{a-1, m+1}, X_{a, m+1}$

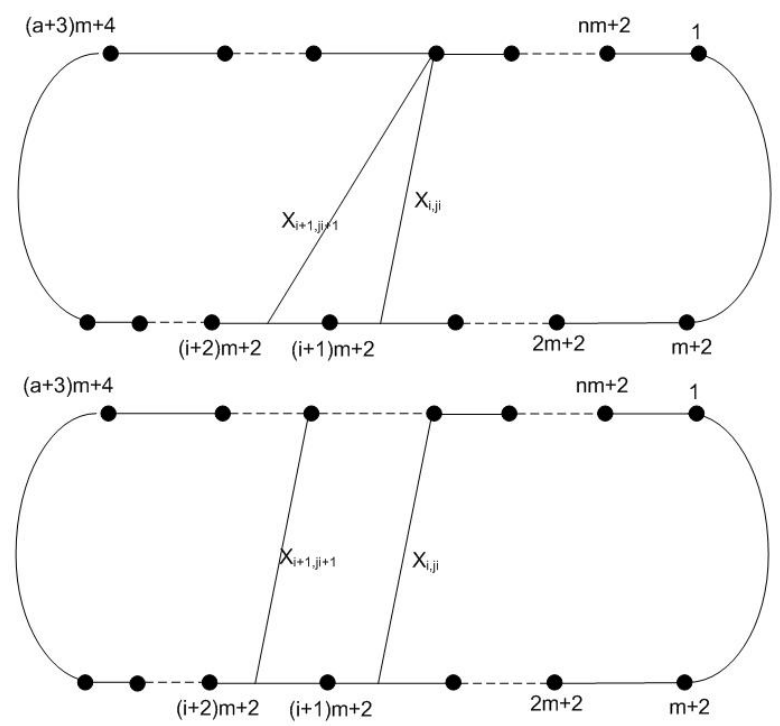

FIGURE $30 . m$-diagonals $X_{i, j_{i}}, X_{i+1, j_{i+1}}$

Note that $m$-diagonals $T_{m+1}, X_{k+1, m+1}, X_{k+2, m+1}, \ldots, X_{a, m+1}, T_{m+2}$ have a common endpoint at $(a+2) m+3$. Therefore there exists a composition of irreducible maps

$$
T_{m+1} \rightarrow X_{k+1, m+1} \rightarrow X_{k+2, m+1} \rightarrow \cdots \rightarrow X_{a, m+1} \rightarrow T_{m+2}
$$


Since there is no other $m$-diagonal whose one endpoint is $(a+2) m+3$ and in the arc $D_{(a+1) m+2,(a+2) m+3}$ then this composition of irreducible maps correspond to

$$
(k+m+1) \stackrel{\alpha_{k+m+1}}{\longrightarrow}(k+m+2) \stackrel{\alpha_{k+m+2}}{\longrightarrow} \cdots \rightarrow(n-2) \stackrel{\alpha_{n-2}}{\longrightarrow}(n-1) \stackrel{\alpha_{n-1}}{\longrightarrow} n .
$$

We conclude that $\rho_{k+m+1}, \rho_{k+m+2}, \ldots, \rho_{n-3}, \rho_{n-2} \notin \mathcal{I}$. The path

$$
(k+m) \stackrel{\alpha_{k+m}}{\longrightarrow}(k+m+1) \stackrel{\alpha_{k+m+1}}{\longrightarrow}(k+m+2)
$$

in $Q$ correspond to the composition of irreducible maps $X \rightarrow T_{m+1} \rightarrow X_{k+1, m+1}$ where $X=T_{m}$ or $X=X_{k, m}$. Because either $m$-diagonals $T_{m}, T_{m+1}, X_{k+1, m+1}$ or $X_{k, m}, T_{m+1}, X_{k+1, m+1}$ always satisfy case 1 in Lemma 2.1 , then $\rho_{k+m} \in \mathcal{I}$.

Example 4.17. Let $m=3$ and $n=7$ then $a=n-m-2=2$ and $m(n+1)+2=26$. The figure of $m$-diagonals that correspond to $T$ in Lemma 4.16 for this case is
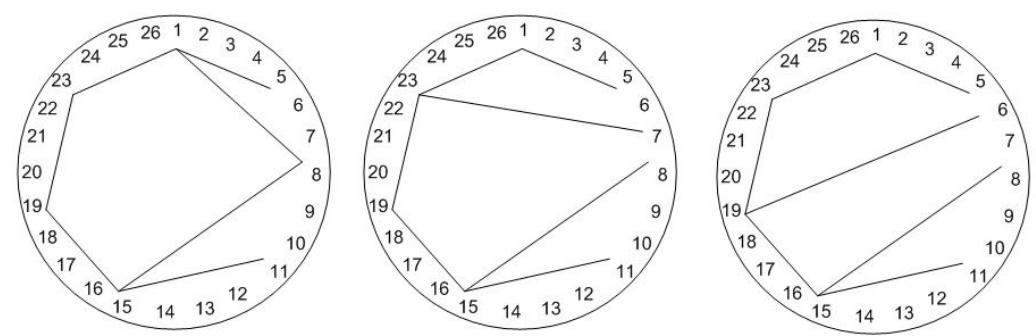

FiguRE 31. $m$-diagonals of $T$ for $m=3$ and $n=7$

Lemma 4.16 gives us the information of $m$-CTA from type $A_{n}$ which is a Nakayama algebra of acyclic type and have $m$ relations. Therefore we can compute the number of $m$-CTA from type $A_{n}$ which has less than or equal to $m$ relations. By the second part of Lemma 4.14, the number of $m$-CTA which have less than $m$ relations of paths of length two is

$$
\left(\begin{array}{c}
n-2 \\
0
\end{array}\right)+\left(\begin{array}{c}
n-2 \\
1
\end{array}\right)+\cdots+\left(\begin{array}{c}
n-2 \\
m-2
\end{array}\right)+\left(\begin{array}{c}
n-2 \\
m-1
\end{array}\right)
$$

Next, the possibility of the number of $m$-CTAs that have exactly $m$ relations of path of length two is $\left(\begin{array}{c}n-2 \\ m\end{array}\right)$. But, by Lemma 4.12 there are $a m$-CTAs who have $m$ relations which are not Nakayama algebras of acyclic type and from Lemma 4.13 we get one more this kind. So the number of $m$-CTAs which have $m$ relations and whose quiver is $A_{n}$ for this case is at most $\left(\begin{array}{c}n-2 \\ m\end{array}\right)-(a+1)$. We compute the number of $m$-cluster tilting objects in Lemma 4.14 part (iii) together with Lemma 4.16 part (ii). Since $1 \leq j_{1} \leq j_{2} \cdots \leq j_{k}<m$ and $j_{k} \neq 1$ then for every $k$ the number of $m$-cluster tilting objects is $\left(\begin{array}{c}m+k \\ k\end{array}\right)-1$. Because $1 \leq k \leq a$ then the 
total number of $m$-cluster tilting objects in Lemma 4.14 part (iii) and Lemma 4.16 part (ii) is

$$
\sum_{k=1}^{a}\left(\begin{array}{c}
m+k \\
k
\end{array}\right)-a
$$

Using Pascal's identity it can be proved that

$$
\sum_{k=0}^{a}\left(\begin{array}{c}
m+k \\
k
\end{array}\right)=\left(\begin{array}{c}
n+a+1 \\
a
\end{array}\right)
$$

We know that $a=n-2-m$, hence

$$
\begin{aligned}
\sum_{k=1}^{a}\left(\begin{array}{c}
m+k \\
k
\end{array}\right)-a & =\sum_{k=1}^{a}\left(\begin{array}{c}
m+k \\
k
\end{array}\right)+1-(a+1) \\
& =\sum_{k=1}^{a}\left(\begin{array}{c}
m+k \\
k
\end{array}\right)+\left(\begin{array}{c}
m+0 \\
0
\end{array}\right)-(a+1) \\
& =\sum_{k=0}^{a}\left(\begin{array}{c}
m+k \\
k
\end{array}\right)-(a+1) \\
& =\left(\begin{array}{c}
m+a+1 \\
a
\end{array}\right)-(a+1) \\
& =\left(\begin{array}{c}
n-2 \\
n-2-m
\end{array}\right)-(a+1) \\
& =\left(\begin{array}{c}
n-2 \\
m
\end{array}\right)-(a+1) .
\end{aligned}
$$

We conclude that all $m$-CTAs which are Nakayama algebras of acyclic type and have $m$ relations of paths of length two are the algebras in Lemma 4.14 part (iii) and Lemma 4.16 part (ii). We write the results so far for the case $m<n-2$ in the following theorem.

Theorem 4.18. Let $H \cong K Q / \mathcal{I}$ be an $m-C T A$ of $\mathcal{C}_{A_{n}}^{m}$ with $m<n-2$, and let $\mathcal{I}$ be an ideal generated by less than or equal to $m$ relations of paths of length two and $Q$ is

$$
1 \stackrel{\alpha_{1}}{\longrightarrow} 2 \stackrel{\alpha_{2}}{\longrightarrow} 3 \rightarrow \cdots \rightarrow(n-1) \stackrel{\alpha_{n-1}}{\longrightarrow} n .
$$

Suppose that $W=\left\{\rho_{j}=\alpha_{j} \alpha_{j+1} \mid 1 \leq j \leq n-2\right\}$ then the generator of $\mathcal{I}$ is one of the following

(i) $B \subseteq W$ for any $B$ with $0 \leq|B|<m$.

(ii) $B \subseteq W$ for any $B$ with $|B|=m$ and $B \neq\left\{\rho_{t}, \rho_{t+1}, \ldots, \rho_{t+m-1}\right\}$ for every $1 \leq t \leq a+1$.

Proof. Apply Lemma 4.11, 4.12, 4.13, 4.14, 4.16.

Until here we have known all $m$-CTAs $H=K Q / \mathcal{I}$ with $Q=A_{n}$ and $I$ generated by at most $m$ relations of path of length two for the case $m<n-2$. 
Next we will give some $m$-CTAs whose ideal is generated by more than $m$ relations of paths of length two.

Proposition 4.19. Suppose that $m<n-2$ and $k m \leq a$ with $1 \leq k \leq(a-1)$ then there is no $m$-cluster tilting object $T$ of $\mathcal{C}_{A_{n}}^{m}$ such that $\operatorname{End}^{o p}(T) \cong \bar{K} Q / \mathcal{I}$ with $Q$ is

$$
1 \stackrel{\alpha_{1}}{\longrightarrow} 2 \stackrel{\alpha_{2}}{\longrightarrow} 3 \rightarrow \cdots \rightarrow(n-1) \stackrel{\alpha_{n-1}}{\longrightarrow} n
$$

and ideal $\mathcal{I}$ generated by at least $(n-2-k)$ paths of length two.

Proof. Assume that such $m$-cluster tilting object $T$ exists. First assume that $a \geq$ $k(m+1)$. Since $1 \leq k \leq(a-1)$ and $\mathcal{I}$ generated by at least $(n-2-k)$ relations of paths of length two then there exist $(m+2) m$-diagonals which configuration is as in Figure 32. Observe that $D_{y_{m+2} x_{1}}$ has at least $(m+2)(m+1)$ sides. Hence

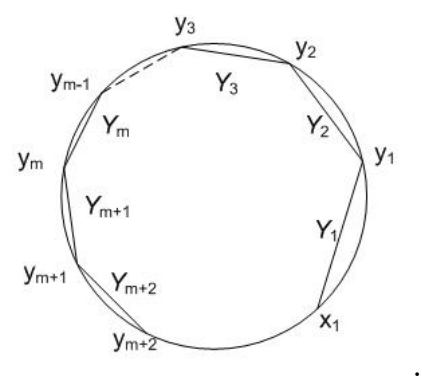

Figure 32. $m$-diagonals $Y_{1}, Y_{2}, \ldots, Y_{m+2}$

$\operatorname{arc} D_{x_{1} y_{m+2}}$ has at least

$$
m(n+1)+2-(m+2)(m+1)=a m
$$

sides. Now there are $a m$-diagonals which are not shown in the Figure 32 . Since $\mathcal{I}$ is generated by at least $(n-2-k)$ paths of length two, there exist $m$-diagonals $X_{1}, X_{2}, \ldots X_{a-k}$ together with $(m+2) m$-diagonal in the Figure 32 such that the configuration as in the Figure 33 . Note that arc $D_{x y_{m+2}}$ at least has $(a-k)(m+1)$ sides. Since $a \geq k(m+1)$ we get

$$
(a-k)(m+1)=a m+(a-k(m+1)) \geq a m,
$$

a contradiction. Now assume that $k m \leq a<k(m+1)$. Consider Figure 32, we obtain that arc $D_{x_{1} x}$ has at least $(k(m+1)-a)$ sides. Hence

$$
k(m+1)-a \leq k(m+1)-k m \leq k .
$$

But there exist $k m$-diagonals of $T$ besides $Y_{1}, Y_{2}, \ldots, Y_{m+1}, Y_{m+2}, X_{1}, X_{2}, \ldots, X_{a-k}$. Each of them has one endpoint outside arc $D_{x_{1} x}$ and the other endpoint should be in arc $D_{x_{1} x}$ and different from $x_{1}, x$. Since arc $D_{x_{1} x}$ has at most $k$ sides then there exist two $m$-diagonals from these $k m$-diagonals whose common endpoint is 


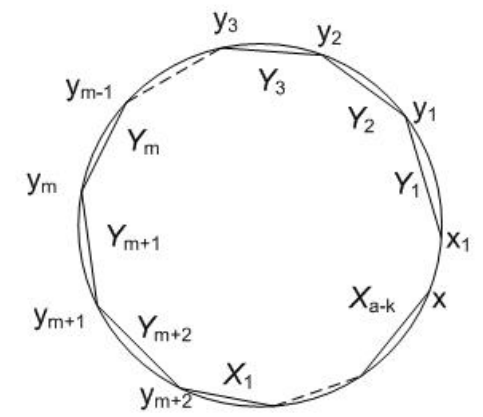

Figure 33. $m$-diagonals $Y_{1}, Y_{2}, \ldots, Y_{m+2}, X_{1}, X_{2}, \ldots X_{a-k}$

in $\operatorname{arc} D x_{1} x$. Consequently the quiver of $\operatorname{End}^{o p}(T)$ has a cycle, a contradiction. This completes the proof.

Consider Proposition 4.19 for the case $k=a-1$. If $k=a-1$ then

$$
a \geq(a-1)(m+1) \Leftrightarrow a \leq 1+\frac{1}{m} .
$$

We get that $a$ must be equal to 1 . If $a=1$ or equivalently $n-2=m+1$ then by Lemma 4.10 the ideal $I$ is generated by at most $m$ relations of paths of length two.

Proposition 4.20. Suppose that $2 \leq m<n-2,1<a=(n-2-m)<m$ and $T=T_{1} \oplus T_{2} \oplus \cdots \oplus T_{m+2} \oplus T_{m+3} \oplus T_{m+4} \oplus \cdots \oplus T_{m+2+t} \oplus X_{1, j_{1}} \oplus X_{2, j_{2}} \oplus \cdots \oplus X_{a-t, j_{a-t}}$ with $1 \leq j_{1} \leq j_{2} \leq \cdots \leq j_{a-t} \leq m+1,1 \leq t \leq a-1$ and $j_{a-t}>t$ then $T$ is an m-cluster tilting object of $\mathcal{C}_{A_{n}}^{m}$.

(i) if $j_{s-1}=1$ and $j_{s}=m+1$ for $1 \leq s \leq a-t$ then the algebra $\operatorname{End}^{o p}(T) \cong$ $K Q / \mathcal{I}$ where $Q$ is

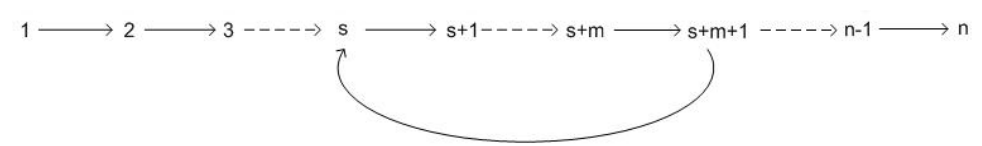

and $\mathcal{I}$ generated by all paths of length two in the cycle and $t$ paths of length two from the right.

(ii) If $j_{a-t}=t+1$ then $\operatorname{End}^{o p}(T) \cong K Q / \mathcal{I}$ where $Q$ is

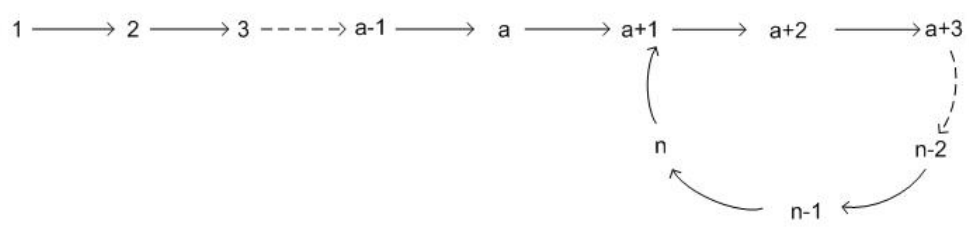


and $\mathcal{I}$ generated by all paths of length two in the cycle and $t$ paths of length two in the path $1 \rightarrow 2 \rightarrow 3 \rightarrow \cdots \rightarrow a \rightarrow a+1$.

(iii) Otherwise $\operatorname{End}^{o p}(T) \cong K Q / \mathcal{I}$ where $Q$ is

$$
1 \stackrel{\alpha_{1}}{\longrightarrow} 2 \stackrel{\alpha_{2}}{\longrightarrow} 3 \rightarrow \cdots \rightarrow(n-1) \stackrel{\alpha_{n-1}}{\longrightarrow} n
$$

and $\mathcal{I}$ generated by $(m+t)$ relations of paths of length two with $\rho_{n-t-1}, \ldots, \rho_{n-3}, \rho_{n-2} \in$ $\mathcal{I}$.

Proof. It is clear that $m$-diagonals which correspond to $T_{1}, T_{2}, \ldots, T_{m+2+t}$ are not crossing each other in $\mathcal{P}_{m(n+1)+2}$. Now consider case (1) that is $j_{s-1}=1$ and $j_{s}=m+1$ for $1 \leq s \leq a-t$. We get that $T=T_{1} \oplus T_{2} \oplus \cdots \oplus T_{m+2+t} \oplus X_{1,1} \oplus$ $X_{2,1} \oplus \cdots \oplus X_{s-1,1} \oplus X_{s, m+1} \oplus X_{s+1, m+1} \oplus \cdots \oplus X_{a-t, m+1}$. We have that

$$
\begin{aligned}
& X_{1,1}=(1,2 m+2) \\
& X_{2,1}=(1,3 m+2) \\
& \vdots \\
& X_{s-1,1}=(1, s m+2) \\
& X_{s, m+1}=(a m+3, s m+2) \\
& X_{s+1, m+1}=(a m+3,(s+1) m+2) \\
& \vdots \\
& X_{a-t, m+1}=(a m+3,(a-t) m+2) \\
& T_{m+2+t}=((a-t) m+m+2-t,(a-t+1) m+m+3-t) .
\end{aligned}
$$

It follows that $X_{s-1,1}$ and $X_{s, m+1}$ have a common endpoint. Since $t \leq a-1<m$ then $m$ diagonals $T_{m+2+t}, X_{a-t, m+1}$ are not crossing each other and do not have a common endpoint. We get the figure of $m$-diagonals which correspond to $T$ for this case is as in Figure 34. Now we come to the case 2, let $j_{a-t}=t+$

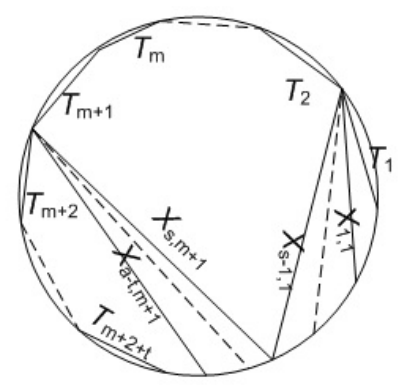

FiguRE 34. $m$-diagonals of $T$

1. Note that $X_{a-t, t+1}=((n-t+1) m+3-t,(n-m-t-1) m+2-t)$ and 
$T_{m+2+t}=((n-m-t-1) m+2-t,(n-m-t) m+3-t)$. It turns out that $X_{a-t, t+1}$ and $T_{m+2+t}$ have a common endpoint and $T_{1}, T_{2}, \ldots, T_{m+2+t}, X_{a-t, t+1}$ are not crossing each other in $\mathcal{P}_{m(n+1)+2}$. We obtain the figure of $m$-diagonals $T_{1}, T_{2}, \ldots, T_{m+2+t}, X_{a-t, t+1}$ in $\mathcal{P}_{m(n+1)+2}$ as in Figure 35 . It is easy to check that

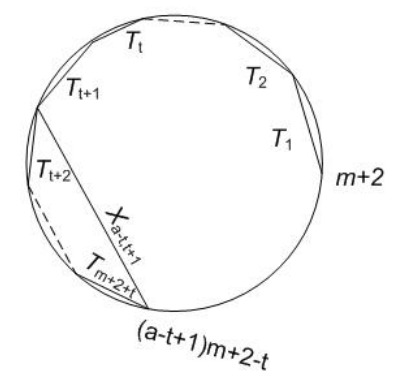

Figure $35 . m$-diagonals $T_{1}, T_{2}, \ldots, T_{m+2}, X_{a-t, t+1}$

$X_{1, j_{t}}, X_{2, j_{2}}, \ldots, X_{a-t-1, j_{a-t-1}}$ are not crossing each other since $t \leq a-1<m$ and $1 \leq j_{1} \leq j_{2} \leq \cdots \leq j_{a-t}=t+1$.

We end the case $m<n-2$ by the above proposition. We have not been able to find all $m$-CTAs which is Nakayama algebra type $A_{n}$. This is because many cases on the value of $a$ have to be considered and have different characteristics in some cases of the value of $a$. However, Proposition 4.19 gives some $m$-CTAs which are not Nakayama algebras in the case $k m \leq a$ with $1 \leq k \leq a-1$. While Proposition 4.20 part (3) give some $m$-CTAs which are Nakayama algebras in the case $1<a<m$ and have more than $m$ relations. A way to find all $m$-CTAs which are Nakayama algebras in this case is by investigating all $m$-CTAs in each case $k m \leq a$ where $1 \leq k \leq a-1$.

Example 4.21. The following figure shows $m$-diagonals correspond to $m$-cluster tilting objects in Proposition 4.20 in the case $m=4$ and $n=9$. 

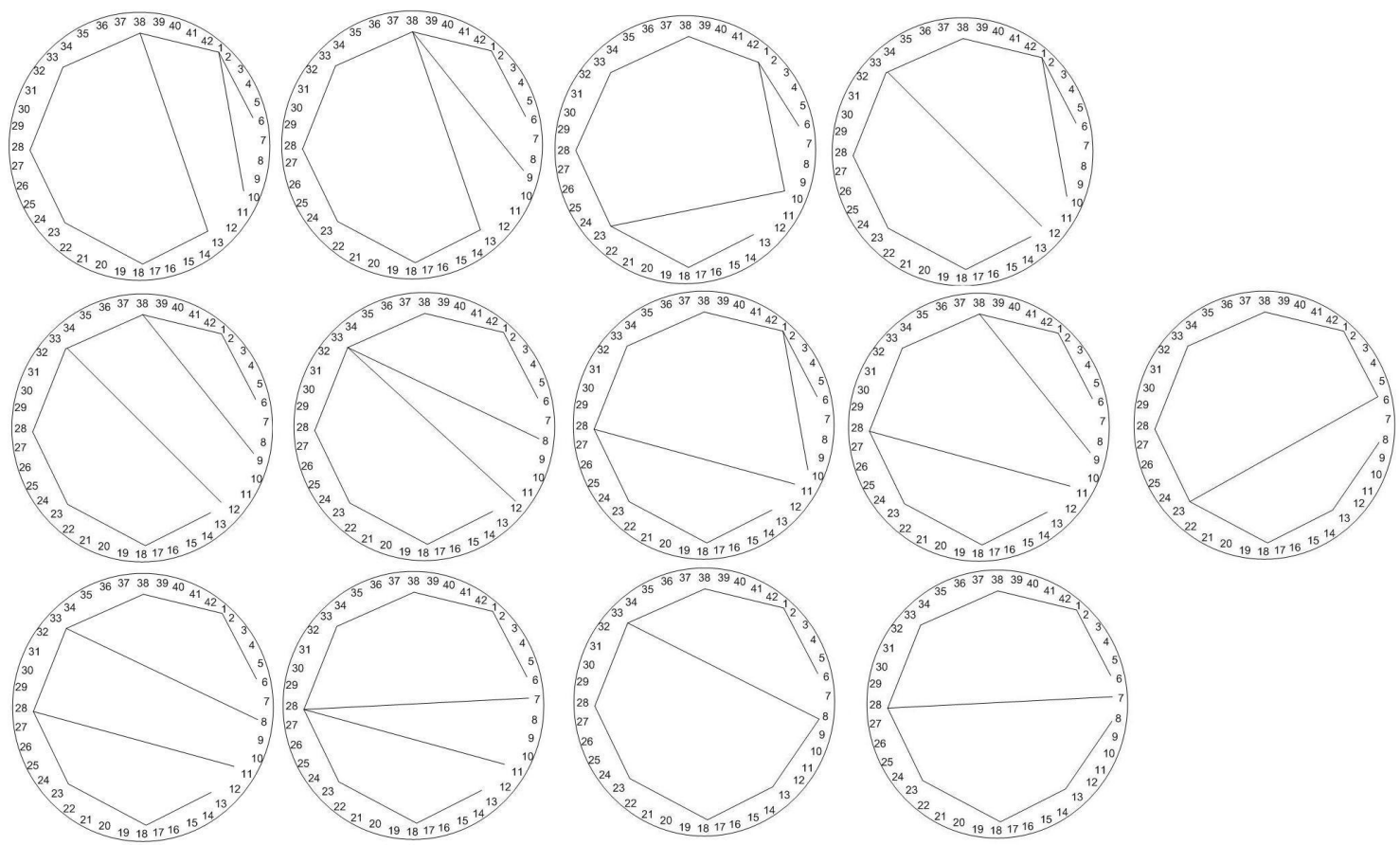

FiguRE 36. $m$-diagonals of $T$ for $m=4$ and $n=9$

Acknowledgement. The first author would like to thank Prof. Aslak Bakke Buan for many helpful discussions while preparing this paper. This research is supported by IMHERE Sandwich Program 2012.

\section{REFERENCES}

[1] Baur, K. and Marsh, R.J., "A Geometric description of $m$-Cluster categories", Trans. Amer. Math. Soc., 360(2008), 5789-5803.

[2] Buan, A., Marsh, R., Reineke, M., Reiten, I., and Todorov, G., "Tilting theory and cluster combinatorics", Advances in mathematics, 204 (2006), 572-68.

[3] Caldero, P., Chapoton, F., and Schiffler, R., "Quivers with relations arising from clusters ( $A_{n}$ case)", Trans. Amer. Math. Soc., 358(2006), no. 3, 1347-1364.

[4] Faisal and Muchtadi-Alamsyah, I., "On cyclic Nakayama $m$-cluster tilted algebra of type $A_{n} "$, Proc. International Conference on Mathematical Research, Education and Application, (2013), 119-127.

[5] Keller, B., "On triangulated orbit categories", Doc. Math., 10(2005), 551-581.

[6] Murphy, G.J., "Derived equivalence classification of $m$-cluster tilted algebra of type $A_{n}$ ", Journal of Algebra, (2009).

[7] Zhu, B., "Generalized cluster complexes via quiver representations", J. Algebraic Combin., $\mathbf{2 7}(2008), 25-54$ 
